\title{
Statistics of cosmic microwave background polarization
}

\author{
Marc Kamionkowski* \\ Department of Physics, Columbia University, 538 West 120th Street, New York, New York 10027
}

Arthur Kosowsky ${ }^{\dagger}$

Harvard-Smithsonian Center for Astrophysics, 60 Garden Street, Cambridge, Massachusetts 02138 and Department of Physics, Lyman Laboratory, Harvard University, Cambridge, Massachusetts 02138

\author{
Albert Stebbins ${ }^{\ddagger}$ \\ NASA/Fermilab Astrophysics Center, Fermi National Accelerator Laboratory, Batavia, Illinois 60510-0500
}

(Received 18 November 1996)

\begin{abstract}
We present a formalism for analyzing a full-sky temperature and polarization map of the cosmic microwave background. Temperature maps are analyzed by expanding over the set of spherical harmonics to give multipole moments of the two-point correlation function. Polarization, which is described by a second-rank tensor, can be treated analogously by expanding in the appropriate tensor spherical harmonics. We provide expressions for the complete set of temperature and polarization multipole moments for scalar and tensor metric perturbations. Four sets of multipole moments completely describe isotropic temperature and polarization correlations; for scalar metric perturbations one set is identically zero, giving the possibility of a clean determination of the vector and tensor contributions. The variance with which the multipole moments can be measured in idealized experiments is evaluated, including the effects of detector noise, sky coverage, and beam width. Finally, we construct coordinate-independent polarization two-point correlation functions, express them in terms of the multipole moments, and derive small-angle limits. [S0556-2821(97)05012-1]

PACS number(s): 98.70.Vc, 98.80.Cq
\end{abstract}

\section{INTRODUCTION}

With the advent of a new generation of balloon-borne and ground-based experiments [1] and satellite missions [2,3], the cosmic microwave background (CMB) will provide an unprecedented window to the early Universe. In addition to determining the origin of large-scale structure, it has been argued that $\mathrm{CMB}$ temperature maps may determine cosmological parameters and the ionization history of the Universe, and perhaps probe long-wavelength gravitational waves [4-9].

Any mechanism which produces temperature anisotropies will invariably lead to polarization as well [10-14]. Temperature fluctuations are the result of perturbations in the gravitational potentials, which contribute directly to the fluctuations via gravitational redshifting (the Sachs-Wolfe effect [15]) and which drive acoustic oscillations of the primordial plasma [5]. These processes result in temperature fluctuations which are the same order of magnitude as the metric perturbations. In contrast, polarization is not directly generated by metric perturbations: a net polarization arises from Compton scattering only when the incident radiation field possesses a nonzero quadrupole moment [16,13], but only monopole and dipole fluctuations are possible as long as the photons in the Universe remain tightly coupled to the charged electrons. Polarization is only generated very near the surface of last scattering as the photons begin to decouple

\footnotetext{
*Electronic address: kamion@phys.columbia.edu

†Electronic address: akosowsky@cfa.harvard.edu

¥Electronic address: stebbins@fnal.gov
}

from the electrons and generate a quadrupole moment through free streaming [17]. Since by this time most of the electrons have recombined into neutral hydrogen, the number of scatterers available to produce polarization is reduced, so $\mathrm{CMB}$ polarization fluctuations are characteristically at a part in $10^{6}$, an order of magnitude below the temperature fluctuations.

A polarization map will provide information that complements that from a temperature map. For example, polarization may help distinguish the gravitational-potential and peculiar-velocity contributions to the acoustic peaks in the temperature-anisotropy power spectrum [11]. In models with reionization, some of the information lost from damping of temperature anisotropies will be regained in the polarization spectrum [18]. Perhaps most importantly, the densityperturbation and gravitational-wave or vorticity contributions to the anisotropy can be geometrically decomposed with a polarization map [16,19-21]. Furthermore, although these nonscalar signals are expected to be small, they will not be swamped by cosmic variance from scalar modes (as discussed further below). Detection of gravity waves is important for testing inflation and for learning about the inflaton potential which drove inflation [22].

Realistically, detection will present a significant experimental challenge. Current results limit the magnitude of linear polarization to roughly a part in $10^{5}$ [23]. Experiments being planned or built will improve sensitivities by at least an order of magnitude [24]. The MAP satellite will make polarized measurements of the entire microwave sky in around a million pixels with a precision of around one part in $10^{5}$ per pixel [2]. If CMB polarization is not discovered by a ground or balloon experiment in the next four years, this 
satellite will almost certainly make the first detection. The Planck Surveyor (formerly COBRAS/SAMBA) may also make polarized measurements [3]. These experimental prospects, as well as the theoretical considerations above, motivate the analysis presented in this paper.

Previous theoretical treatments of CMB polarization have relied on a small-angle approximation, which is valid when considering patches of the sky small enough to be approximated as flat. Upcoming polarization maps will require a more sophisticated formalism. In this paper, we develop in detail a description of polarization on the full sky. The Stokes parameters conventionally used to describe polarization are not invariant under rotations of the coordinate system used to describe them, unlike temperature fluctuations, but rather transform as a second-rank tensor [13]. By expressing the polarization in terms of a complete, orthonormal set of tensor basis functions on the celestial sphere, power spectra and correlation functions which are independent of the coordinate system can be constructed. Earlier work on small patches of the sky chose a particular reference coordinate system which completely defines the polarization but obscures the physical interpretation of the polarization pattern. Also, the signal from vector and tensor perturbations is expected to contribute to $\mathrm{CMB}$ polarization primarily at large angles on the sky through gravitational effects, so the correct full-sky analysis is essential.

Our formalism is stated in terms of differential geometry on the sphere, using a notation widely used in general relativity. Similar calculations have recently been performed by Seljak and Zaldarriaga [20,21], using spin-weighted spherical harmonics [25]. Although the formalisms employed differ substantially and the calculations are quite lengthy, we have verified that the end results are equivalent where they overlap, giving us confidence both are correct.

After a brief review of Stokes parameters, the next section defines the tensor spherical harmonic basis functions and gives useful explicit expressions and formulas for decomposing a polarization map into its harmonic components. Section III covers the statistics of the expansion coefficients of the temperature and polarization harmonics, derivations of variance estimates for the various multipole moments in idealized experiments, and a recipe for simulating a combined polarization and temperature map given theoretical angular power spectra. Section IV derives exact expressions for all of the multipole moments from scalar and tensor metric fluctuations, expressed in terms of the conventional Fourier components of radiation brightnesses. Section V then treats twopoint correlation functions of the Stokes parameters in a coordinate-independent manner and expresses the multipole moments and correlation functions in terms of each other. We also reproduce flat-sky results by taking small-angle limits and make an explicit connection with earlier work in particular fixed coordinate systems. Finally, a summary and discussion section briefly considers detection prospects for various polarization signals. A pair of mathematical appendixes collect results from differential geometry on the sphere and useful identities of Legendre polynomials and spherical harmonics.

\section{DESCRIPTION OF POLARIZATION}

\section{A. Review of Stokes parameters}

The cosmic microwave background is characterized completely by its temperature and polarization in each direction on the sky (assuming its frequency spectrum is a perfect blackbody). Polarized radiation is described in terms of the Stokes parameters $Q, U$, and $V$ [26]. For a monochromatic electromagnetic wave of frequency $\omega_{0}$ propagating in the $z$ direction, the components of the wave's electric field vector at a given point in space can be written as

$$
E_{x}=a_{x}(t) \cos \left[\omega_{0} t-\theta_{x}(t)\right], \quad E_{y}=a_{y}(t) \cos \left[\omega_{0} t-\theta_{y}(t)\right] .
$$

If these two components are correlated, then the wave is said to be polarized. The Stokes parameters are defined as the time averages

$$
\begin{gathered}
I \equiv\left\langle a_{x}^{2}\right\rangle+\left\langle a_{y}^{2}\right\rangle, \\
Q \equiv\left\langle a_{x}^{2}\right\rangle-\left\langle a_{y}^{2}\right\rangle, \\
U \equiv\left\langle 2 a_{x} a_{y} \cos \left(\theta_{x}-\theta_{y}\right)\right\rangle, \\
V \equiv\left\langle 2 a_{x} a_{y} \sin \left(\theta_{x}-\theta_{y}\right)\right\rangle .
\end{gathered}
$$

The parameter $I$ gives the radiation intensity which is positive definite. The other three parameters can take either sign and describe the polarization state. For unpolarized radiation, $Q=U=V=0$. The Stokes parameters are additive for incoherent superpositions of waves, which makes them natural variables for describing polarized radiative transport.

In most applications polarization is measured in units of intensity; however it is conventional and convenient when studying the CMB to express polarization in terms of the difference in brightness temperature of a particular polarization state from that of the mean brightness temperature of the $\mathrm{CMB}$. The rationale for this convention comes from the well-known result that the spectrum of polarization induced in the CMB is exactly the same as a temperature anisotropy, so in brightness temperature units the polarization should be independent of frequency.

The Stokes parameters $I$ and $V$ describe physical observables and are independent of the choice of coordinate system. However, $Q$ and $U$ describe orthogonal modes of linear polarization and depend on the axes in relation to which the linear polarization is defined. From Eqs. (2.5), it is easy to show that when the coordinate system is rotated by an angle $\alpha$, the same radiation field is now described by the parameters

$$
\begin{gathered}
Q^{\prime}=Q \cos (2 \alpha)+U \sin (2 \alpha), \\
U^{\prime}=-Q \sin (2 \alpha)+U \cos (2 \alpha) .
\end{gathered}
$$

Stated another way, under rotations of the coordinate system around the direction of propagation, the $Q$ and $U$ Stokes parameters transform like the independent components of a two-dimensional, second rank symmetric trace-free (STF) tensor. Thus we can equally well describe the linear polarization state by a polarization tensor $\mathcal{P}_{a b}$, which coincides with the photon density matrix [13].

\section{B. Scalar and tensor harmonic expansions}

Suppose we have an all-sky map of the CMB temperature $T(\hat{\mathbf{n}})$ and polarization tensor $\mathcal{P}_{a b}(\hat{\mathbf{n}})$. The polarization tensor 
is a $2 \times 2$ symmetric $\left(\mathcal{P}_{a b}=\mathcal{P}_{b a}\right)$ and trace-free $\left(g^{a b} \mathcal{P}_{a b}=0\right)$ tensor, so it is specified by two real quantities. Given the Stokes parameters $Q$ and $U$ measured in any coordinate system, we can construct $\mathcal{P}_{a b}$. For example, in spherical polar coordinates $(\theta, \phi)$, the metric is $g_{a b}=\operatorname{diag}\left(1, \sin ^{2} \theta\right)$ and

$$
\mathcal{P}_{a b}(\hat{\mathbf{n}})=\frac{1}{2}\left(\begin{array}{cc}
Q(\hat{\mathbf{n}}) & -U(\hat{\mathbf{n}}) \sin \theta \\
-U(\hat{\mathbf{n}}) \sin \theta & -Q(\hat{\mathbf{n}}) \sin ^{2} \theta
\end{array}\right) .
$$

The factors of $\sin \theta$ must be included since the coordinate basis for $(\theta, \phi)$ is an orthogonal, but not an orthonormal basis. (For more details of differential geometry on the twosphere, see Appendix A.) The Compton scattering process which thermalizes the $\mathrm{CMB}$ and generates polarization cannot produce any net circular polarization [27]; thus we expect $V=0$ for the microwave background and do not consider the $V$ Stokes parameter further. Note the spherical polar coordinate system adopted in this paper gives an outward direction for the $z$ axis, which is opposite the radiation propagation direction. The convention with the $z$ axis in the direction of propagation is sometimes used, particularly in Ref. [13]; this leads to the opposite sign for the $U$ Stokes parameter, but all results are unchanged.

In the usual way, we can expand the temperature pattern $T(\hat{\mathbf{n}})$ in a set of complete orthonormal basis functions, the spherical harmonics

$$
\frac{T(\hat{\mathbf{n}})}{T_{0}}=1+\sum_{l=1}^{\infty} \sum_{m=-l}^{l} a_{(l m)}^{\mathrm{T}} Y_{(l m)}(\hat{\mathbf{n}})
$$

where

$$
a_{(l m)}^{\mathrm{T}}=\frac{1}{T_{0}} \int d \hat{\mathbf{n}} T(\hat{\mathbf{n}}) Y_{(l m)}^{*}(\hat{\mathbf{n}})
$$

are the temperature multipole coefficients and $T_{0}$ is the mean CMB temperature. The $l=1$ term in Eq. (2.8) is indistinguishable from the kinematic dipole and is normally ignored.

Similarly, we can expand the polarization tensor in terms of a complete set of orthonormal basis functions for symmetric trace-free $2 \times 2$ tensors on the two-sphere:

$$
\frac{\mathcal{P}_{a b}(\hat{\mathbf{n}})}{T_{0}}=\sum_{l=2}^{\infty} \sum_{m=-l}^{l}\left[a_{(l m)}^{\mathrm{G}} Y_{(l m) a b}^{\mathrm{G}}(\hat{\mathbf{n}})+a_{(l m)}^{\mathrm{C}} Y_{(l m) a b}^{\mathrm{C}}(\hat{\mathbf{n}})\right]
$$

where the expansion coefficients are given by

$$
\begin{aligned}
& a_{(l m)}^{\mathrm{G}}=\frac{1}{T_{0}} \int d \hat{\mathbf{n}} \mathcal{P}_{a b}(\hat{\mathbf{n}}) Y_{(l m)}^{\mathrm{G} a b} *(\hat{\mathbf{n}}), \\
& a_{(l m)}^{\mathrm{C}}=\frac{1}{T_{0}} \int d \hat{\mathbf{n}} \mathcal{P}_{a b}(\hat{\mathbf{n}}) Y_{(l m)}^{\mathrm{C} a b} *(\hat{\mathbf{n}}),
\end{aligned}
$$

which follow from the orthonormality properties

$$
\begin{aligned}
& \int d \hat{\mathbf{n}} Y_{(l m) a b}^{\mathrm{G} *}(\hat{\mathbf{n}}) Y_{\left(l^{\prime} m^{\prime}\right)}^{\mathrm{G} a b}(\hat{\mathbf{n}})=\int d \hat{\mathbf{n}} Y_{(l m) a b}^{\mathrm{C} *}(\hat{\mathbf{n}}) Y_{\left(l^{\prime} m^{\prime}\right)}^{\mathrm{C} a b}(\hat{\mathbf{n}}) \\
& =\delta_{l l^{\prime}} \delta_{m m^{\prime}} \\
& \int d \hat{\mathbf{n}} Y_{(l m) a b}^{\mathrm{G} *}(\hat{\mathbf{n}}) Y_{\left(l^{\prime} m^{\prime}\right)}^{\mathrm{C} a b}(\hat{\mathbf{n}})=0
\end{aligned}
$$

Note that unlike scalar harmonics, the tensor harmonics only exist for $l \geqslant 2$ [28].

The basis functions $Y_{(l m) a b}^{\mathrm{G}}(\hat{\mathbf{n}})$ and $Y_{(l m) a b}^{\mathrm{C}}(\hat{\mathbf{n}})$ are given in terms of covariant derivatives of the spherical harmonics by [28]

$$
Y_{(l m) a b}^{\mathrm{G}}=N_{l}\left(Y_{(l m): a b}-\frac{1}{2} g_{a b} Y_{(l m): c} c\right)
$$

and

$$
Y_{(l m) a b}^{\mathrm{C}}=\frac{N_{l}}{2}\left(Y_{(l m): a c} \epsilon_{b}^{c}+Y_{(l m): b c} \epsilon_{a}^{c}\right)
$$

where $\epsilon_{a b}$ is the completely antisymmetric tensor, the : denotes covariant differentiation on the two-sphere, and

$$
N_{l} \equiv \sqrt{\frac{2(l-2) !}{(l+2) !}}
$$

is a normalization factor.

The existence of two sets of basis functions, labeled here by $\mathrm{G}$ and $\mathrm{C}$, is due to the fact that a symmetric trace-free (STF) $2 \times 2$ tensor is specified by two independent parameters. In two dimensions, any STF tensor can be uniquely decomposed into a part of the form $A_{: a b}-(1 / 2) g_{a b} A_{: c}{ }^{c}$ and another part of the form $B_{a c} \epsilon^{c}{ }_{b}+B_{: b c} \epsilon_{a}^{c}$, where $A$ and $B$ are two scalar functions. This decomposition is quite similar to the decomposition of a vector field into a part which is the gradient of a scalar field and a part which is the curl of a vector field; hence we use the notation G for "gradient"' and C for "curl." Since the $Y_{(l m)}$ 's provide a complete basis for scalar functions on the sphere, the $Y_{(l m) a b}^{\mathrm{G}}$ and $Y_{(l m) a b}^{\mathrm{C}}$ tensors provide a complete basis for G-type and C-type STF tensors, respectively. This G-C decomposition is also known as the scalar-pseudoscalar decomposition [28].

Incidentally, these tensor spherical harmonics are identical to those which appear in the theory of gravitational radiation $[29,30]$. The propagating degrees of freedom of gravitational field perturbations are described by a spin-2 tensor. Computing the flux of gravitational radiation from a source requires the components of the gravitational field tangent to a sphere around the source which are induced by the motions of that source. Our G harmonics are often [29] — but not always [30]—referred to as having "electric-type" parity, since an electric field can be written as the gradient of a scalar. Likewise, our C harmonics have "magnetic-type" parity since they are the curl of a vector field. The two varieties of harmonics also correspond to electric and magnetic multipole radiation.

Integration by parts transforms Eqs. (2.11) into integrals over scalar spherical harmonics and derivatives of the polarization tensor: 


$$
\begin{gathered}
a_{(l m)}^{\mathrm{G}}=\frac{N_{l}}{T_{0}} \int d \hat{\mathbf{n}} Y_{(l m)}^{*}(\hat{\mathbf{n}}) \mathcal{P}_{a b}: a b(\hat{\mathbf{n}}), \\
a_{(l m)}^{\mathrm{C}}=\frac{N_{l}}{T_{0}} \int d \hat{\mathbf{n}} Y_{(l m)}^{*}(\hat{\mathbf{n}}) \mathcal{P}_{a b}: a c(\hat{\mathbf{n}}) \epsilon_{c}{ }^{b},
\end{gathered}
$$

where the second equation uses the fact that $\epsilon^{a b}: c=0$. These forms are useful for theoretical calculations of the multipole moments. We don't recommend taking second derivatives of real data. Since $T$ and $\mathcal{P}_{a b}$ are real, all of the multipole must obey the reality condition

$$
a_{(l m)}^{\mathrm{X} *}=(-1)^{m} a_{(l,-m)}^{\mathrm{X}},
$$

where $X=\{T, G, C\}$.

\section{Explicit form of the harmonics}

In spherical polar coordinates $(\theta, \phi)$ the tensor spherical harmonics are given explicitly by $[28,30]$

$$
Y_{(l m) a b}^{\mathrm{G}}(\hat{\mathbf{n}})=\frac{N_{l}}{2}\left(\begin{array}{cc}
W_{(l m)}(\hat{\mathbf{n}}) & X_{(l m)}(\hat{\mathbf{n}}) \sin \theta \\
X_{(l m)}(\hat{\mathbf{n}}) \sin \theta & -W_{(l m)}(\hat{\mathbf{n}}) \sin ^{2} \theta
\end{array}\right)
$$

and

$$
Y_{(l m) a b}^{\mathrm{C}}(\hat{\mathbf{n}})=\frac{N_{l}}{2}\left(\begin{array}{cc}
-X_{(l m)}(\hat{\mathbf{n}}) & W_{(l m)}(\hat{\mathbf{n}}) \sin \theta \\
W_{(l m)}(\hat{\mathbf{n}}) \sin \theta & X_{(l m)}(\hat{\mathbf{n}}) \sin ^{2} \theta
\end{array}\right),
$$

where

$$
\begin{aligned}
W_{(l m)}(\hat{\mathbf{n}}) & =\left(\frac{\partial^{2}}{\partial \theta^{2}}-\cot \theta \frac{\partial}{\partial \theta}+\frac{m^{2}}{\sin ^{2} \theta}\right) Y_{(l m)}(\hat{\mathbf{n}}) \\
& =\left(2 \frac{\partial^{2}}{\partial \theta^{2}}-l(l+1)\right) Y_{(l m)}(\hat{\mathbf{n}})
\end{aligned}
$$

and

$$
X_{(l m)}(\hat{\mathbf{n}})=\frac{2 i m}{\sin \theta}\left(\frac{\partial}{\partial \theta}-\cot \theta\right) Y_{(l m)}(\hat{\mathbf{n}}) .
$$

(Note that this definition of $X_{(\operatorname{lm})}(\hat{\mathbf{n}})$ differs from that in Ref. [30] by a factor of $\sin \theta$.) The exchange symmetry $\{Q, U\} \leftrightarrow\{U,-Q\} \quad$ as $\mathrm{G} \leftrightarrow \mathrm{C}$ indicates that $Y_{(\operatorname{lm}) a b}^{\mathrm{G}}$ and $Y_{(l m) a b}^{\mathrm{C}}$ represent polarizations rotated by $45^{\circ}$. By evaluating the derivatives, these functions can be written

$$
\begin{gathered}
W_{(l m)}(\hat{\mathbf{n}})=2 \sqrt{\frac{2 l+1}{4 \pi} \frac{(l-m) !}{(l+m) !}} G_{(l m)}^{+}(\cos \theta) e^{i m \phi}, \\
i X_{(l m)}(\hat{\mathbf{n}})=-2 \sqrt{\frac{2 l+1}{4 \pi} \frac{(l-m) !}{(l+m) !}} G_{(l m)}^{-}(\cos \theta) e^{i m \phi},
\end{gathered}
$$

where the real functions $G_{(l m)}^{ \pm}$are defined by [28]

$$
\begin{aligned}
G_{(l m)}^{+}(\cos \theta) \equiv & -\left(\frac{l-m^{2}}{\sin ^{2} \theta}+\frac{1}{2} l(l-1)\right) P_{l}^{m}(\cos \theta) \\
& +(l+m) \frac{\cos \theta}{\sin ^{2} \theta} P_{l-1}^{m}(\cos \theta), \\
G_{(l m)}^{-}(\cos \theta) \equiv & \frac{m}{\sin ^{2} \theta}\left((l-1) \cos \theta P_{l}^{m}(\cos \theta)\right. \\
& \left.-(l+m) P_{l-1}^{m}(x)\right) .
\end{aligned}
$$

These expressions will be useful for the correlation functions in Sec. V, and for simulating maps and data analysis.

In linear theory, scalar perturbations can produce only G-type polarization and not C-type polarization. On the other hand, tensor or vector metric perturbations will produce a mixture of both types [19,20,31]. Heuristically, this is because scalar perturbations have no handedness so they cannot produce any "curl,", whereas vector and tensor perturbations do have a handedness and therefore can. Observation of a nonzero primordial component of C-type polarization (a nonzero $\left.a_{(l m)}^{\mathrm{C}}\right)$ in the $\mathrm{CMB}$ would provide compelling evidence for significant contribution of either vector or tensor perturbations at the time of last scattering.

Given a polarization map of even a small part of the sky, one could in principle test for vector or tensor contribution by computing the combination of derivatives of the polarization field given by $\mathcal{P}^{a b}{ }_{a b c} \epsilon^{c}{ }_{a}$ which will be nonzero only for C-type polarization. Of course, taking derivatives of noisy data is problematic. We discuss more robust probes of this signal below.

\section{STATISTICS OF THE MULTIPOLE COEFFICIENTS}

\section{A. Statistical independence of the coefficients}

We now have three sets of multipole moments, $a_{(l m)}^{\mathrm{T}}$, $a_{(l m)}^{\mathrm{G}}$, and $a_{(l m)}^{\mathrm{C}}$, which fully describe the temperature or polarization map of the sky. Statistical isotropy implies that

$$
\left\langle a_{(l m)}^{\mathrm{T} *} a_{\left(l^{\prime} m^{\prime}\right)}^{\mathrm{T}}\right\rangle=C_{l}^{\mathrm{T}} \delta_{l l^{\prime}} \delta_{m m^{\prime}}, \quad\left\langle a_{(l m)}^{\mathrm{G} *} a_{\left(l^{\prime} m^{\prime}\right)}^{\mathrm{G}}\right\rangle=C_{l}^{\mathrm{G}} \delta_{l l^{\prime}} \delta_{m m^{\prime}},
$$

$\left\langle a_{(l m)}^{\mathrm{C} *} a_{\left(l^{\prime} m^{\prime}\right)}^{\mathrm{C}}\right\rangle=C_{l}^{\mathrm{C}} \delta_{l l^{\prime}} \delta_{m m^{\prime}}, \quad\left\langle a_{(l m)}^{\mathrm{T} *} a_{\left(l^{\prime} m^{\prime}\right)}^{\mathrm{G}}\right\rangle=C_{l}^{\mathrm{TG}} \delta_{l l^{\prime}} \delta_{m m^{\prime}}$,

$$
\left\langle a_{(l m)}^{\mathrm{T} *} a_{\left(l^{\prime} m^{\prime}\right)}^{\mathrm{C}}\right\rangle=C_{l}^{\mathrm{TC}} \delta_{l l^{\prime}} \delta_{m m^{\prime}}
$$$$
\left\langle a_{(l m)}^{\mathrm{G} *} a_{\left(l^{\prime} m^{\prime}\right)}^{\mathrm{C}}\right\rangle=C_{l}^{\mathrm{GC}} \delta_{l l^{\prime}} \delta_{m m^{\prime}}
$$

where the angle brackets are an average over all realizations. For Gaussian theories, the statistical properties of a temperature or polarization map are specified fully by these six sets of multipole moments. In fact, the scalar spherical harmonics $Y_{(l m)}$ and the $\mathrm{G}$ tensor harmonics $Y_{(l m) a b}^{\mathrm{G}}$ have parity $(-1)^{l}$, but the $\mathrm{C}$ harmonics $Y_{(l m) a b}^{\mathrm{C}}$ have parity $(-1)^{l+1}$. Therefore, symmetry under parity transformations requires that $C_{l}^{\mathrm{TC}}=C_{l}^{\mathrm{GC}}=0$, which will also be demonstrated explicitly in the following section. Measurement of nonzero cosmological values for these moments would be quite extraordinary, demonstrating a handedness to primordial perturbations. In practice, these two sets of moments can be used to monitor foreground emission. Furthermore, as men- 
tioned above and demonstrated explicitly in Sec. IV, $C_{l}^{\mathrm{C}}=0$ for scalar metric perturbations $[19,20]$. At small angular scales where the contribution from tensor and vector perturbations is expected to be negligible, $C_{l}^{\mathrm{C}}$ can also be pressed into duty as a foreground monitor. Exact expressions for these multipole moments in terms of the photon brightnesses usually calculated by early-Universe Boltzmann codes are derived below.

\section{B. Map simulation}

For the case of Gaussian statistics, realizations of temperature or polarizations maps are easy to generate using standard techniques. Since the only cross correlation between mode coefficients, given by $C_{l}^{\mathrm{TG}}$, correlates only $a_{(l m)}^{\mathrm{T}}$ and $a_{(l m)}^{\mathrm{G}}$ with the same $l$ and $m$, the total correlation matrix is block diagonal with the largest blocks being only $2 \times 2$ matrices. In particular, set

$$
\begin{gathered}
a_{(l m)}^{\mathrm{T}}=\zeta_{1}\left(C_{l}^{\mathrm{T}}\right)^{1 / 2}, \\
a_{(l m)}^{\mathrm{G}}=\zeta_{1} \frac{C_{l}^{\mathrm{TG}}}{\left(C_{l}^{\mathrm{T}}\right)^{1 / 2}}+\zeta_{2}\left(C_{l}^{\mathrm{G}}-\frac{\left(C_{l}^{\mathrm{TG}}\right)^{2}}{C_{l}^{\mathrm{T}}}\right)^{1 / 2}, \\
a_{(l m)}^{\mathrm{C}}=\zeta_{3}\left(C_{l}^{\mathrm{C}}\right)^{1 / 2},
\end{gathered}
$$

where for each value of $l$ and $m>0$ choose three complex numbers $\left(\zeta_{1}, \zeta_{2}, \zeta_{3}\right)$ drawn from a Gaussian distribution with unit variance, i.e., both $\sqrt{2} \operatorname{Re}\left(\zeta_{i}\right)$ and $\sqrt{2} \operatorname{Im}\left(\zeta_{i}\right)$ are drawn from a normal distribution. For $m=0$ the same equations hold but the $\zeta_{i}$ should be real and normally distributed; for $m<0$ the coefficients are given by Eq. (2.19). Note that in all cases $C_{l}^{\mathrm{G}} C_{l}^{\mathrm{T}} \geqslant\left(C_{l}^{\mathrm{TG}}\right)^{2}$. This set of coefficients can be combined with Eqs. (2.7), (2.10), (2.23), and (2.22) to obtain the explicit expressions

$$
\begin{aligned}
Q(\hat{\mathbf{n}}) & =2 \mathcal{P}_{\theta \theta}(\hat{\mathbf{n}}) \\
& =T_{0} \sum_{l=2}^{\infty} \sum_{m=-l}^{l} N_{l}\left[a_{(l m)}^{\mathrm{G}} W_{(l m)}(\hat{\mathbf{n}})-a_{(l m)}^{\mathrm{C}} X_{(l m)}(\hat{\mathbf{n}})\right], \\
U(\hat{\mathbf{n}}) & =-2 \csc \theta \mathcal{P}_{\theta \phi}(\hat{\mathbf{n}}) \\
& =-T_{0} \sum_{l=2}^{\infty} \sum_{m=-l}^{l} N_{l}\left[a_{(l m)}^{\mathrm{G}} X_{(l m)}(\hat{\mathbf{n}})+a_{(l m)}^{\mathrm{C}} W_{(l m)}(\hat{\mathbf{n}})\right]
\end{aligned}
$$

with $W_{(l m)}$ and $X_{(l m)}$ given by Eqs. (2.24) and (2.25). Note that polarization maps are traditionally plotted as headless vectors with amplitude $\left(Q^{2}+U^{2}\right)^{1 / 2}$ and orientation angle $(1 / 2) \arctan (U / Q)$.

\section{Estimators}

One of the the main uses of a temperature/polarization map will be to determine the multipole moments with the best possible accuracy. From a full-sky CMB temperature map, we can construct the following rotationally invariant estimators (denoted by a caret) for the multipole coefficients

$$
\begin{gathered}
\widehat{C_{l}^{\mathrm{T}}}=\sum_{m=-l}^{l} \frac{\left|a_{(l m)}^{\mathrm{T}}\right|^{2}}{2 l+1}, \quad \widehat{C_{l}^{\mathrm{G}}}=\sum_{m=-l}^{l} \frac{\left|a_{(l m)}^{\mathrm{G}}\right|^{2}}{2 l+1}, \\
\widehat{C_{l}^{\mathrm{C}}}=\sum_{m=-l}^{l} \frac{\left|a_{(l m)}^{\mathrm{C}}\right|^{2}}{2 l+1}, \quad \widehat{C_{l}^{\mathrm{TG}}}=\sum_{m=-l}^{l} \frac{a_{(l m)}^{\mathrm{T} *} a_{(l m)}^{\mathrm{G}}}{2 l+1} .
\end{gathered}
$$

Note that Eq. (2.19) guarantees that the $\widehat{C_{l}^{\mathrm{TG}}}$ will be real.

When averaged over the sky (denoted by an overbar), the mean square temperature anisotropy after subtracting the dipole is

$$
\overline{\left(\frac{\Delta T}{T_{0}}\right)^{2}}=\sum_{l=2}^{\infty} \frac{2 l+1}{4 \pi} \widehat{C_{l}^{\mathrm{T}}}
$$

and the mean square polarization is

$$
\begin{gathered}
\overline{\mathcal{P}^{2}} \equiv \overline{Q^{2}+U^{2}}=2 \overline{\mathcal{P}^{a b} \mathcal{P}_{a b}}=\overline{\mathcal{P}_{\mathrm{G}}^{2}}+\overline{\mathcal{P}_{\mathrm{C}}^{2}}, \\
\overline{\mathcal{P}_{\mathrm{G}}{ }^{2}} \equiv T_{0}^{2} \sum_{l=2}^{\infty} \frac{2 l+1}{8 \pi} \widehat{C_{l}^{\mathrm{G}}}, \quad \overline{\mathcal{P}_{\mathrm{C}}^{2}} \equiv T_{0}^{2} \sum_{l=2}^{\infty} \frac{2 l+1}{8 \pi} \widehat{C_{l}^{\mathrm{C}}} .
\end{gathered}
$$

Even if no single $\widehat{C_{l}^{\mathrm{C}}}$ or $\widehat{C_{l}^{\mathrm{G}}}$ gives a significant signal, combining different $l$ 's as in $\overline{\mathcal{P}_{\mathrm{G}}{ }^{2}}$ or $\overline{\mathcal{P}_{\mathrm{C}}{ }^{2}}$ can give a statistically significant signal.

\section{Cosmic and pixel-noise variance}

The averages in Eqs. (3.1) are over an ensemble of universes drawn from a theoretically defined statistical distribution, or assuming ergodicity, a spatial average over all observer positions in the Universe. However, we can only observe a single realization of the ensemble from a single location. Therefore, even if we had an ideal (full-sky coverage, no foreground contamination, infinite angular resolution, and no instrumental noise) experiment, the accuracy with which the estimators in Eqs. (3.4) could recover the multipole moments would be limited by a sample variance known as "cosmic variance." Furthermore, a realistic experiment may have limited sky coverage and angular resolution and some instrumental noise. In this section, we calculate the cosmic variance with which the multipole moments can be recovered. We also calculate the variance due to finite sky coverage, angular resolution, and instrumental noise in an idealized experiment. To do so, we adopt a simplified model in which we assume a pixelized map in which the noise in each pixel is independent and Gaussian distributed after foregrounds have been successfully subtracted. In many respects, our derivation follows that in Ref. [32], and our results agree with those in Ref. [21].

We must first determine the contribution of pixel noise to each multipole moment, and we begin with the temperature moments. Consider a temperature map of the full sky $T^{\text {map }}(\hat{\mathbf{n}})$, which is pixelized with $N_{\text {pix }}$ pixels. If we assume that each pixel subtends the same area on the sky then we 
can construct multipole coefficients of the temperature map using

$$
\begin{aligned}
d_{(l m)}^{\mathrm{T}} & =\int d \hat{\mathbf{n}}\left(\frac{T^{\mathrm{map}}(\hat{\mathbf{n}})}{T_{0}}\right) Y_{(l m)}(\hat{\mathbf{n}}) \\
& \simeq \frac{1}{T_{0}} \sum_{j=1}^{N_{\text {pix }}} \frac{4 \pi}{N_{\text {pix }}} T_{j}^{\mathrm{map}} Y_{(l m)}\left(\hat{\mathbf{n}}_{j}\right),
\end{aligned}
$$

where $T_{j}^{\text {map }}$ is the measured temperature perturbation in pixel $j$ and $\hat{\mathbf{n}}_{j}$ is its direction. The difference between $d_{(l m)}$ and $a_{(l m)}$ is that the former includes the effects of finite beam size and detector noise; i.e., $d_{(l m)}$ is measured. The extent to which the approximate equality fails is the pixelization noise, which is small on angular scales much larger than the pixels. In many of the proposed experiments, which oversample the sky when compared to their beam, there will be no loss of information by ignoring scales close to the pixel scale. The observed temperature is due to a cosmological signal and a pixel noise, $T_{j}^{\text {map }}=T_{j}+T_{j}^{\text {noise }}$. If we assume that each pixel has the same rms noise, and that the noise in each pixel is uncorrelated with that in any other pixel, and is uncorrelated with the cosmological signal, $i . e ., \quad\left\langle T_{i}^{\text {noise }} T_{j}^{\text {noise }}\right\rangle$ $=T_{0}^{2}\left(\sigma_{\text {pix }}^{\mathrm{T}}\right)^{2} \delta_{i j}$ and $\left\langle T_{i} T_{j}^{\text {noise }}\right\rangle=0$, then

$$
\begin{aligned}
\left\langle d_{(l m)}^{\mathrm{T}} d_{\left(l^{\prime} m^{\prime}\right)}^{\mathrm{T} *}\right\rangle & =\left\langle a_{(l m)}^{\mathrm{T}} a_{\left(l^{\prime} m^{\prime}\right)}^{\mathrm{T} *}\right\rangle+\left\langle a_{(l m)}^{\mathrm{T}, \text { noise }}\left(a_{\left(l^{\prime} m^{\prime}\right)}^{\mathrm{T}, \text { noise }}\right)^{*}\right\rangle \\
& =\left|W_{l}^{\mathrm{b}}\right|^{2} C_{l}^{\mathrm{T}} \delta_{l l^{\prime}} \delta_{m m^{\prime}}+\left\langle a_{(l m)}^{\mathrm{T}, \text { noise }}\left(a_{\left(l^{\prime} m^{\prime}\right)}^{\mathrm{T}, \text { noise }}\right) *\right\rangle,
\end{aligned}
$$

where we have written the expectation value of the cosmological signal in terms of that predicted by theory $C_{l}^{\mathrm{T}}$ multiplied by $\left|W_{l}^{b}\right|^{2}$ which accounts for beam smearing. Typically the beam is approximately Gaussian in shape, corresponding to the window function $W_{l}^{\mathrm{b}} \approx \exp \left(-l^{2} \sigma_{\mathrm{b}}^{2} / 2\right)$, where $\sigma_{\mathrm{b}}=\theta_{\mathrm{FWHM}} / \sqrt{8 \ln 2}=0.00742\left(\theta_{\mathrm{FWHM}} / 1^{\circ}\right)$ and $\theta_{\mathrm{FWHM}}$ gives the full-width at half maximum.

The second term in Eq. (3.9) is

$$
\begin{aligned}
\left\langle a_{(l m)}^{\mathrm{T}, \text { noise }}\left(a_{\left(l^{\prime} m^{\prime}\right)}^{\mathrm{T}, \text { noise }}\right) *\right\rangle= & \frac{1}{T_{0}^{2}} \sum_{i=1}^{N_{\text {pix }}} \sum_{j=1}^{N_{\text {pix }}}\left(\frac{4 \pi}{N_{\text {pix }}}\right)^{2}\left\langle T_{i}^{\text {noise }} T_{j}^{\text {noise }}\right\rangle \\
& \times Y_{(l m)}\left(\hat{\mathbf{n}}_{i}\right) Y_{\left(l^{\prime} m^{\prime}\right)}^{*}\left(\hat{\mathbf{n}}_{j}\right) \\
= & \left(\sigma_{\text {pix }}^{\mathrm{T}}\right)^{2}\left(\sum_{i=1}^{N_{\text {pix }}} \frac{4 \pi}{N_{\text {pix }}} Y_{(l m)}\left(\hat{\mathbf{n}}_{i}\right)\right. \\
& \left.\times Y_{\left(l^{\prime} m^{\prime}\right)}\left(\hat{\mathbf{n}}_{j}\right)\right) \frac{4 \pi}{N_{\text {pix }}} \\
= & \frac{4 \pi\left(\sigma_{\text {pix }}^{\mathrm{T}}\right)^{2}}{N_{\text {pix }}} \delta_{l l^{\prime}} \delta_{m m^{\prime}} .
\end{aligned}
$$

Therefore, the moments measured by the map are distributed with a variance [32]

$$
\left\langle d_{(l m)}^{\mathrm{T}} d_{\left(l^{\prime} m^{\prime}\right)}^{\mathrm{T} *}\right\rangle=\left(C_{l}\left|W_{l}^{\mathrm{b}}\right|^{2}+\frac{4 \pi\left(\sigma_{\mathrm{pix}}^{\mathrm{T}}\right)^{2}}{N_{\mathrm{pix}}}\right) \delta_{l l^{\prime}} \delta_{m m^{\prime}} .
$$

Now we move on to the noise contribution to the polarization moments. We will assume the instrumental noise in the polarization measurements is isotropic, the same for all pixels, uncorrelated with the noise in the anisotropy, which in terms of the Stokes parameters requires

$$
\begin{gathered}
\left\langle Q_{i}^{\text {noise }} Q_{j}^{\text {noise }}\right\rangle=\left\langle U_{i}^{\text {noise }} U_{j}^{\text {noise }}\right\rangle=T_{0}^{2}\left(\sigma_{\text {pix }}^{\mathrm{P}}\right)^{2} \delta_{i j}, \\
\left\langle Q_{i}^{\text {noise }} U_{j}^{\text {noise }}\right\rangle=\left\langle Q_{i}^{\text {noise }} T_{j}^{\text {noise }}\right\rangle=\left\langle U_{i}^{\text {noise }} T_{j}^{\text {noise }}\right\rangle=0 .
\end{gathered}
$$

We denote the polarization tensor describing the noise in pixel $i$ by $\mathcal{P}_{a b}^{\text {noise }}\left(\hat{\mathbf{n}}_{i}\right)$. The previous equations are equivalent to the coordinate-independent equation (see Ref. [28])

$$
\begin{gathered}
\left\langle\mathcal{P}_{a b}^{\text {noise }}\left(\hat{\mathbf{n}}_{i}\right) \mathcal{P}_{c d}^{\text {noise }}\left(\hat{\mathbf{n}}_{j}\right)\right\rangle=\frac{1}{4} T_{0}^{2}\left(\sigma_{\text {pix }}^{\mathrm{P}}\right)^{2}\left(g_{a c} g_{b d}-\epsilon_{a c} \epsilon_{b d}\right) \delta_{i j} \\
\left\langle\mathcal{P}_{a b}^{\text {noise }}\left(\hat{\mathbf{n}}_{i}\right) T^{\text {noise }}\left(\hat{\mathbf{n}}_{j}\right)\right\rangle=0
\end{gathered}
$$

The mode coefficients for the noise, defined as in Eq. (2.11), will have a correlation matrix

$$
\begin{aligned}
\left\langle a_{(l m)}^{\mathrm{X}, \text { noise }}\right. & \left.a_{\left(l^{\prime} m^{\prime}\right)}^{\mathrm{X}^{\prime} \text {,noise } *}\right\rangle \\
= & \left(\frac{4 \pi}{N_{\mathrm{pix}} T_{0}}\right)^{2} \sum_{i=1}^{N_{\mathrm{pix}}} \sum_{j=1}^{N_{\mathrm{pix}}} Y_{(l m)}^{\mathrm{X} a b} *\left(\hat{\mathbf{n}}_{i}\right) Y_{\left(l^{\prime} m^{\prime}\right)}^{\mathrm{X}^{\prime} c d}\left(\hat{\mathbf{n}}_{j}\right) \\
& \times\left\langle\mathcal{P}_{a b}\left(\hat{\mathbf{n}}_{i}\right) \mathcal{P}_{c d}\left(\hat{\mathbf{n}}_{j}\right)\right\rangle, \quad \mathrm{X}, \mathrm{X}^{\prime} \in\{\mathrm{G}, \mathrm{C}\}
\end{aligned}
$$

so using Eqs. (3.13), (2.13), and (A8) we find

$$
\begin{aligned}
& \left\langle a_{(l m)}^{\mathrm{X}, \text { noise }} a_{\left(l^{\prime} m^{\prime}\right)}^{\mathrm{X}^{\prime} \text {, noise } *}\right\rangle \\
& =\frac{1}{4}\left(\frac{4 \pi \sigma_{\mathrm{pix}}^{\mathrm{P}}}{N_{\mathrm{pix}}}\right)^{2} \sum_{i=1}^{N_{\mathrm{pix}}} 2 Y_{(l m)}^{\mathrm{X} a b} *\left(\hat{\mathbf{n}}_{i}\right) Y_{\left(l^{\prime} m^{\prime}\right) a b}^{\mathrm{X}^{\prime}}\left(\hat{\mathbf{n}}_{i}\right) \\
& =\frac{2 \pi}{N_{\text {pix }}}\left(\sigma_{\mathrm{pix}}^{\mathrm{P}}\right)^{2} \delta_{l l^{\prime}} \delta_{m m^{\prime}} \delta_{\mathrm{XX}}
\end{aligned}
$$

and, of course,

$$
\left\langle a_{(l m)}^{\mathrm{G}, \text { noise }} * a_{\left(l^{\prime} m^{\prime}\right)}^{\mathrm{T}, \text { noise }}\right\rangle=\left\langle a_{(l m)}^{\mathrm{C} \text {, noise }} * a_{\left(l^{\prime} m^{\prime}\right)}^{\mathrm{T}, \mathrm{noise}}\right\rangle=0 .
$$

Thus instrumental noise contributes equally to the measured G- and C-polarization components but introduces no cross correlation between them.

Collecting the results, if $d_{(l m)}^{\mathrm{X}}$ (for $\mathrm{X}=\{\mathrm{T}, \mathrm{G}, \mathrm{C}\}$ ) are the multipole coefficients for the map (signal plus noise), their variances will be

$$
\begin{aligned}
\left\langle d_{(l m)}^{\mathrm{X} *} d_{\left(l^{\prime} m^{\prime}\right)}^{\mathrm{X}^{\prime}}\right\rangle & =\left(\left|W_{l}^{\mathrm{b}}\right|^{2} C_{l}^{\mathrm{XX}}+w_{\mathrm{XX}^{\prime}}^{-1}\right) \delta_{l l^{\prime}} \delta_{m m^{\prime}} \\
& \equiv D_{l}^{\mathrm{XX}{ }^{\prime}} \delta_{l l^{\prime}} \delta_{m m^{\prime}}
\end{aligned}
$$

where

$$
w_{\mathrm{T}}^{-1} \equiv \frac{4 \pi\left(\sigma_{\mathrm{pix}}^{\mathrm{T}}\right)^{2}}{N_{\mathrm{pix}}}, \quad w_{\mathrm{GG}}^{-1}=w_{\mathrm{CC}}^{-1} \equiv w_{\mathrm{P}}^{-1} \equiv \frac{2 \pi\left(\sigma_{\mathrm{pix}}^{\mathrm{P}}\right)^{2}}{N_{\mathrm{pix}}},
$$


and $w_{\mathrm{XX}^{\prime}}^{-1}=0$ for $\mathrm{X} \neq \mathrm{X}^{\prime}$. The quantities $w^{-1}$ are inverse statistical weights per unit solid angle, a measure of experimental sensitivity independent of pixel size [32]. (Note that our $w_{\mathrm{P}}^{-1}$ differs by a factor of two from that in Ref. [21] which is consistent with our $C_{l}^{\mathrm{G}}$ and $C_{l}^{\mathrm{C}}$ differing from their $\mathrm{E}$ and $\mathrm{B}$ moments by a factor of two.)

Estimators for the multipole moments $\mathrm{CMB}$ power spec$\operatorname{tra} C_{l}^{\mathrm{XX}}$ are

$$
C_{l}^{\widehat{\mathrm{XX}}}=\left(\widehat{D_{l}^{\mathrm{XX}}}-w_{\mathrm{XX}^{\prime}}^{-1}\right)\left|W_{l}^{\mathrm{b}}\right|^{-2},
$$

where

$$
\widehat{D_{l}^{\mathrm{XX}}}=\sum_{m=-l}^{l} \frac{d_{(l m)}^{\mathrm{X} *} d_{(l m)}^{\mathrm{X}^{\prime}}}{2 l+1}
$$

Since the estimators for each $D_{l}^{\mathrm{XX}^{\prime}}$ (and therefore for $C_{l}^{\mathrm{XX}^{\prime}}$ ) are constructed from only $2 l+1$ multipole coefficients, each $C_{l}^{\mathrm{XX}}$ can be recovered only with a finite sampling variance, known as cosmic variance. In addition, the six different sets of measured moments are constructed from the three sets of $d_{(l m)}$ coefficients, leading to some covariance between the moments. These variances can be described with a $(6 \times 6)$ covariance matrix

$$
\begin{aligned}
\Xi_{\mathrm{AA}^{\prime}} & \equiv\left\langle\left(\widehat{C_{l}^{\mathrm{A}}}-C_{l}^{\mathrm{A}}\right)\left(\widehat{C_{l}^{\mathrm{A}}}-C_{l}^{\mathrm{A}^{\prime}}\right)\right\rangle=\left\langle\widehat{C_{l}^{\mathrm{A}}} \widehat{C_{l}^{\mathrm{A}}}\right\rangle-C_{l}^{\mathrm{A}} C_{l}^{\mathrm{A}^{\prime}} \\
& =\left(\left\langle\widehat{D_{l}^{\mathrm{A}}} \widehat{D_{l}^{\mathrm{A}}}\right\rangle-D_{l}^{\mathrm{A}} D_{l}^{\mathrm{A}^{\prime}}\right)\left|W_{l}^{\mathrm{b}}\right|^{-4}
\end{aligned}
$$

for $\mathrm{A}=\mathrm{XX}$.

We now calculate the entries of this matrix. Recall that if $x_{i}$ are Gaussian random variables with variances $\left\langle x_{i} x_{j}\right\rangle=\sigma_{i j}^{2}$, then $\left\langle x_{i}^{2} x_{j}^{2}\right\rangle=\sigma_{i i}^{2} \sigma_{j j}^{2}+2 \sigma_{i j}^{2}$, and $\left\langle x_{i}^{3} x_{j}\right\rangle$ $=3 \sigma_{i i}^{2} \sigma_{i j}^{2}$. For $\mathrm{X}=\{\mathrm{T}, \mathrm{G}, \mathrm{C}\}$,

$$
\begin{aligned}
\left\langle\widehat{D_{l}^{\mathrm{XX}}} \widehat{D_{l}^{\mathrm{X}^{\prime} \mathrm{X}}}\right\rangle= & \sum_{m m^{\prime}} \frac{\left\langle\left|d_{(l m)}^{\mathrm{X}}\right|^{2}\left|d_{\left(l m^{\prime}\right)}^{\mathrm{X}^{\prime}}\right|^{2}\right\rangle}{(2 l+1)^{2}} \\
= & \sum_{m m^{\prime}} \frac{1}{(2 l+1)^{2}}\left\{D_{l}^{\mathrm{XX}} D_{l}^{\mathrm{X}^{\prime} \mathrm{X}^{\prime}}\left(1-\delta_{m m^{\prime}}\right)\right. \\
& \left.+\left[D_{l}^{\mathrm{XX}} D_{l}^{\mathrm{X}^{\prime} \mathrm{X}^{\prime}}+2\left(D_{l}^{\mathrm{XX}^{\prime}}\right)^{2}\right] \delta_{m m^{\prime}}\right\} \\
= & D_{l}^{\mathrm{XX}} D_{l}^{\mathrm{XX}}+\frac{2}{(2 l+1)^{2}}\left(D_{l}^{\mathrm{XX}^{\prime}}\right)^{2}
\end{aligned}
$$

The diagonal elements for TT, GG, and CC are thus

$$
\Xi_{\mathrm{XX}, \mathrm{XX}}=\frac{2}{2 l+1}\left(C_{l}^{\mathrm{XX}}+w_{\mathrm{X}}^{-1}\left|W_{l}^{\mathrm{b}}\right|^{-2}\right)^{2}
$$

and the off-diagonal elements are

$$
\begin{gathered}
\Xi_{\mathrm{TT}, \mathrm{GG}}=\frac{2}{2 l+1}\left(C_{l}^{\mathrm{TG}}\right)^{2}, \\
\Xi_{\mathrm{TT}, \mathrm{CC}}=0, \\
\Xi_{\mathrm{GG}, \mathrm{CC}}=0 .
\end{gathered}
$$

For the diagonal TG component,

$$
\begin{aligned}
\left\langle\widehat{D_{l}^{\mathrm{TG}}} \widehat{D_{l}^{\mathrm{TG}}}\right\rangle= & \sum_{m m^{\prime}} \frac{\left\langle d_{(l m)}^{\mathrm{T} *} d_{(l m)}^{\mathrm{G}} d_{\left(l m^{\prime}\right)}^{\mathrm{T} *} d_{\left(l m^{\prime}\right)}^{\mathrm{G}}\right\rangle}{(2 l+1)^{2}} \\
= & \frac{1}{(2 l+1)^{2}} \sum_{m m^{\prime}}\left[\left\langle\left|d_{(l m)}^{\mathrm{T}}\right|^{2}\left|d_{(l m)}^{\mathrm{G}}\right|^{2}\right\rangle \delta_{m m^{\prime}}\right. \\
& \left.+\left\langle d_{(l m)}^{\mathrm{T} *} d_{(l m)}^{\mathrm{G}} d_{\left(l m^{\prime}\right)}^{\mathrm{T}} d_{\left(l m^{\prime}\right)}^{\mathrm{G} *}\right\rangle\left(1-\delta_{m m^{\prime}}\right)\right] \\
= & \sum_{m} \frac{D_{l}^{\mathrm{TT}} D_{l}^{\mathrm{GG}}+2\left(D_{l}^{\mathrm{TG}}\right)^{2}}{(2 l+1)^{2}} \\
& +\sum_{m m^{\prime}} \frac{\left(1-\delta_{m m^{\prime}}\right)\left(D_{l}^{\mathrm{TG}}\right)^{2}}{(2 l+1)^{2}}=\frac{1}{2 l+1}\left[\left(D_{l}^{\mathrm{TG}}\right)^{2}\right. \\
& \left.+D_{l}^{\mathrm{TT}} D_{l}^{\mathrm{TG}}\right]+\left(D_{l}^{\mathrm{TG}}\right)^{2} .
\end{aligned}
$$

Therefore,

$$
\begin{aligned}
\Xi_{\mathrm{TG}, \mathrm{TG}}= & \frac{1}{2 l+1}\left[\left(C_{l}^{\mathrm{TG}}\right)^{2}+\left(C_{l}^{\mathrm{T}}+w_{\mathrm{T}}^{-1}\left|W_{l}^{\mathrm{b}}\right|^{-2}\right)\right. \\
& \left.\times\left(C_{l}^{\mathrm{G}}+w_{\mathrm{P}}^{-1}\left|W_{l}^{\mathrm{b}}\right|^{-2}\right)\right] .
\end{aligned}
$$

The diagonal covariance-matrix elements for TC and TG are obtained similarly and are nonzero even though $C_{l}^{\mathrm{TC}}=C_{l}^{\mathrm{GC}}=0$. Given a map, it should be checked for consistency that there is no statistically significant parity violation.

Finally, for the off-diagonal TG-TT component,

$$
\begin{aligned}
\left\langle\widehat{D_{l}^{\mathrm{TG}}} \widehat{D_{l}^{\mathrm{TT}}}\right\rangle= & \sum_{m m^{\prime}} \frac{1}{(2 l+1)^{2}}\left[\left\langle\left|d_{(l m)}^{\mathrm{T}}\right|^{3} d_{(l m)}^{\mathrm{G}}\right\rangle \delta_{m m^{\prime}}\right. \\
& \left.+\left\langle\left|d_{(l m)}^{\mathrm{T}}\right|^{2}\left(d_{\left(l m^{\prime}\right)}^{\mathrm{T}}\right) * d_{\left(l m^{\prime}\right)}^{\mathrm{G}}\right\rangle\left(1-\delta_{m m^{\prime}}\right)\right] \\
= & \left(\frac{2}{2 l+1}+1\right) D_{l}^{\mathrm{TT}} D_{l}^{\mathrm{TG}} .
\end{aligned}
$$

Therefore,

$$
\Xi_{\mathrm{TT}, \mathrm{TG}}=\frac{2}{2 l+1}\left[C_{l}^{\mathrm{TG}}\left(C_{l}^{\mathrm{T}}+w_{\mathrm{T}}^{-1}\left|W_{l}^{\mathrm{b}}\right|^{-2}\right)\right]
$$

and, similarly,

$$
\Xi_{\mathrm{GG}, \mathrm{TG}}=\frac{2}{2 l+1}\left[C_{l}^{\mathrm{TG}}\left(C_{l}^{\mathrm{G}}+w_{\mathrm{G}}^{-1}\left|W_{l}^{\mathrm{b}}\right|^{-2}\right)\right]
$$

and this completes the calculation of all nonzero elements of the covariance matrix.

To determine the precision with which a temperature or polarization map can recover some cosmological parameters, e.g., $\mathbf{s}=\left\{\Omega_{0}, \Lambda, h, \Omega_{b}, \ldots\right\}$, we can evaluate the curvature, or Fisher information, matrix [33], which can be generalized from that in Ref. [7] to

$$
\alpha_{i j}=\sum_{l} \sum_{\mathrm{A}, \mathrm{A}^{\prime}} \frac{\partial C_{l}^{\mathrm{A}}}{\partial s_{i}}\left[\Xi^{-1}\right]_{\mathrm{AA}^{\prime}} \frac{\partial C_{l}^{\mathrm{A}^{\prime}}}{\partial s_{j}},
$$


for $\mathrm{A}=\mathrm{TT}, \mathrm{GG}, \mathrm{CC}, \mathrm{TG}$, where $\left[\Xi^{-1}\right]_{\mathrm{AA}^{\prime}}$ are elements of the inverse of $\Xi$. The standard error with which a given parameter $s_{i}$ can be recovered after marginalizing over all other parameters is given by the square root of the diagonal element $i$ of $[\alpha]^{-1}$, assuming a linear dependence of $C_{l}^{\mathrm{X}}$ on all of the parameters.

The $w_{\mathrm{X}}^{-1}$ factors on the right-hand side of Eqs. (3.23), (3.26), and (3.29) are those due to instrumental noise. However, note that even in an ideal experiment with $\sigma_{\text {pix }}=0$, the right-hand sides would still be nonzero, and this is the cosmic variance. Equations (3.23), (3.26), and (3.29) are valid for a map with full-sky coverage. Realistically, however, only a fraction $f_{\text {sky }}$ of the sky will be surveyed, or if the entire sky is surveyed, only a fraction will be used in the analysis. Therefore, the accuracy with which cosmological parameters can be recovered will be degraded accordingly. Strictly speaking, harmonic analysis on a cut sky will have to be performed, and this will introduce correlations in the errors of multipole moments of different $l$ 's. However, if the entire sky is surveyed, but only a fraction of the sky is used for the analysis (e.g., if the Galactic plane has been cut out), then the effect of partial sky coverage can be approximated by multiplying the curvature matrix by $f_{\text {sky }}$ (which will increase the standard errors in cosmological parameters by $f_{\text {sky }}^{-1 / 2}$ ). If only a fraction of the sky is surveyed, the curvature matrix should still be multiplied by $f_{\text {sky }}$, but note that $N_{\text {pix }}$ is the number of pixels actually in the map.

\section{E. Pixel noise for a polarization map}

How are the temperature and polarization pixel noises related? If the two linear polarization states are always given equal integration times, the total number of photons available for the temperature measurement will be twice the number available for either polarization measurement. Therefore,

$$
\left(\sigma_{\text {pix }}^{\mathrm{T}}\right)^{2}=\frac{1}{2}\left(\sigma_{\text {pix }}^{\mathrm{P}}\right)^{2} .
$$

However, a crucial difference in overall sensitivity exists between the two current receiver technologies. Coherent receivers [i.e., high-electron-mobility transistor (HEMT) amplifiers] measure the incoming electric field, while incoherent receivers (i.e., bolometers) measure only the incoming total power. In the former case, the signal can be split into two orthogonal linear polarizations and the phase information can be retained throughout the entire signal path. If properly designed, such a system of receivers can measure the linear polarizations without compromising the temperature measurement, so the temperature sensitivity will be determined solely by the amplifier characteristics [2]. This is the design strategy for the MAP satellite.

On the other hand, bolometers measure only the instantaneous total power received and do not retain any phase information; a filter must be placed in front of the detector for each linear polarization state, discarding half of the incoming photons. Thus the temperature sensitivity for a polarized bolometer measurement is only half of that for an unpolarized measurement, which collects twice as many photons in the same amount of integration time. A compensating factor is that bolometers offer much greater raw sensitivity than
HEMT amplifiers. An important question facing future bolometer experiments is whether to sacrifice half of the temperature sensitivity for polarization information [34]. If the goal of an experiment is to measure angular power spectra and the temperature measurements are dominated by cosmic variance, then polarizing the measurement is obviously advantageous. The answer is as yet unclear in cases where cosmic variance is not the controlling factor in the temperature measurement.

\section{CALCULATION OF THE MOMENTS}

In this section, we calculate the set of multipole moments defined by Eqs. (3.1), for scalar and tensor metric perturbations. Vector metric perturbations make a negligible contribution for inflationary theories, although they are generic in defect models; they will be covered elsewhere. The intensity and linear-polarization state of the CMB in any given direction is specified by three quantities (the temperature $T$ and the Stokes parameters $Q$ and $U$ ), giving six possible sets of multipole moments, $C_{l}^{\mathrm{T}}, C_{l}^{\mathrm{G}}, C_{l}^{\mathrm{C}}, C_{l}^{\mathrm{TG}}, C_{l}^{\mathrm{TC}}$, and $C_{l}^{\mathrm{GC}}$, but as argued above, parity demands that $C_{l}^{\mathrm{TC}}=C_{l}^{\mathrm{GC}}=0$.

One way to calculate the moments is to rewrite the radiative transfer equations in terms of tensor harmonics [31]. The contribution of each Fourier mode to each multipole moment is then obtained by evolving numerically the coupled Einstein and Boltzmann equations for the multipole coefficients $a_{(l m)}^{\mathrm{X}}$. Integrating over all Fourier modes then gives the multipole moments. This approach has the advantage of being similar in form to the usual moment hierarchy formulation of the problem, while keeping the independent modes separated throughout the calculation, giving simple power spectrum expressions. A second approach offering computational advantages has been presented in Ref. [21], which uses the Stokes parameter evolution equations to write an integral equation solution for the multipole moments.

Another possibility, which makes contact with previous work on CMB temperature anisotropies and polarization, is to express the multipole moments in terms of the usual perturbations to the photon brightness and polarization $\Delta_{I l}$ and $\Delta_{Q l}$ (see Ref. [13] for definitions and descriptions) obtained from most current numerical calculations [35], and this is the approach adopted here. In this section, we calculate the contributions to these moments from scalar and tensor perturbations. With these results, it is straightforward to modify existing numerical codes to obtain all of the multipole moments.

\section{A. Scalar metric perturbations}

The simplest calculation of the multipole moments in terms of the photon brightnesses uses the fact that, due to statistical isotropy, the contribution of a given $\mathbf{k}$ mode to the moments $C_{l}$ depends on its magnitude only and not its direction. Therefore, we will consider the contribution of a single $\mathbf{k}$ mode, with the coordinate system always chosen with $\hat{\mathbf{z}}$ in the $\mathbf{k}$ direction, and then integrate over all $\mathbf{k}$ at the end. All temperatures, Stokes parameters, polarization tensors, and expansion coefficients are functions of $\mathbf{k}$, although we sometimes drop explicitly references to $\mathbf{k}$ for notational simplicity. Functions of the real-space coordinate $\mathbf{x}$ do not appear in this paper. 


\section{Temperature moments}

We begin with the familiar temperature moments. The temperature anisotropy induced in the direction $\hat{\mathbf{n}}$ on the sky by a single k scalar mode is given by Eq. (7.1) in Ref. [13],

$$
\begin{aligned}
\frac{T(\mathbf{k}, \hat{\mathbf{n}})}{T_{0}} & =\frac{1}{4} \sum_{j=1}^{\infty}(2 j+1) P_{j}(\hat{\mathbf{k}} \cdot \hat{\mathbf{n}}) \Delta_{I j}^{\mathrm{s}}(k) \\
& =1+\sum_{l=2}^{\infty} \sum_{m=-l}^{l} a_{(l m)}^{\mathrm{T}, \mathrm{s}}(\mathbf{k}) Y_{(l m)}(\hat{\mathbf{n}}) .
\end{aligned}
$$

The superscripts $\mathrm{T}$ and $\mathrm{s}$ indicate that we are dealing here with temperature moments from scalar perturbations. The $\Delta_{I l}^{\mathrm{s}}(k)$ are Legendre coefficients of the photon intensity distribution function for scalar metric perturbations [13]. The expansion coefficients are given by the inverse transform, Eq. (2.9),

$$
\begin{aligned}
a_{(l m)}^{\mathrm{T}, \mathrm{s}}(\mathbf{k}) & =\frac{1}{4} \sum_{j=0}^{\infty}(2 j+1) \Delta_{I j}(k) \int d \hat{\mathbf{n}} P_{j}(\hat{\mathbf{k}} \cdot \hat{\mathbf{n}}) Y_{(l m)}^{*}(\hat{\mathbf{n}}) \\
& =\pi \Delta_{I l}^{\mathrm{s}}(k) Y_{(l m)}^{*}(\hat{\mathbf{k}})=\frac{1}{2} \sqrt{(2 l+1) \pi} \Delta_{I l}^{\mathrm{s}}(k) \delta_{m 0},
\end{aligned}
$$

where we have used Eq. (B12) and taking $\hat{\mathbf{k}}=\hat{\mathbf{z}}$ in the final line. The contribution to $C_{l}^{\mathrm{T}, \mathrm{s}}$ from this mode is then

$$
C_{l}^{\mathrm{T}, \mathrm{s}}(\mathbf{k})=\frac{1}{2 l+1} \sum_{m=-l}^{l}\left|a_{(l m)}^{\mathrm{T}, \mathrm{s}}(\mathbf{k})\right|^{2}=\frac{\pi}{4}\left|\Delta_{I l}^{\mathrm{s}}(k)\right|^{2} .
$$

The total multipole moment from scalar modes $C_{l}^{\mathrm{T}, \text { scalar }}$ is given by integrating over all $\mathbf{k}$ :

$$
C_{l}^{\mathrm{T}, \text { scalar }}=\int \frac{d^{3} \mathbf{k}}{(2 \pi)^{3}} C_{l}^{\mathrm{T}, \mathrm{s}}(\mathbf{k})=\frac{1}{8 \pi} \int k^{2} d k\left|\Delta_{I l}^{\mathrm{s}}(k)\right|^{2},
$$

which is the usual result.

\section{Polarization moments}

Now we move on to the polarization moments produced by scalar perturbations. First we describe the "standard", representation of polarization, which is what has usually been computed by Boltzmann codes. In the spherical polar coordinates $(\theta, \phi)$, the Stokes parameters induced in direction $\hat{\mathbf{n}}$ on the sky by a single $\mathbf{k}$ scalar mode in the $\hat{\mathbf{z}}$ direction can be obtained from Eq. (7.4) in Ref. [13] (the $\xi^{\prime}$ there can be chosen zero for $\hat{\mathbf{k}}=\hat{\mathbf{z}}$ ) and are

$$
\frac{Q(\mathbf{k}, \hat{\mathbf{n}})}{T_{0}}=\frac{1}{4} \sum_{j=0}^{\infty}(2 j+1) P_{j}(\hat{\mathbf{k}} \cdot \hat{\mathbf{n}}) \Delta_{Q j}^{\mathrm{s}}(k), \quad U(\mathbf{k}, \hat{\mathbf{n}})=0,
$$

where the $\Delta_{O l}^{\mathrm{s}}(k)$ are Legendre coefficients of the photon polarization distribution function for scalar metric perturbations. The polarization tensor at a point $\hat{\mathbf{n}}$ on the sky induced by this scalar mode is thus

$$
\begin{aligned}
\frac{\mathcal{P}_{a b}(\mathbf{k}, \hat{\mathbf{n}})}{T_{0}} & =\frac{1}{8} \sum_{j=0}^{\infty}(2 j+1) P_{j}(\hat{\mathbf{k}} \cdot \hat{\mathbf{n}}) \Delta_{Q j}^{\mathrm{s}}(k)\left(\begin{array}{cc}
1 & 0 \\
0 & -\sin ^{2} \theta
\end{array}\right) \\
& =\frac{1}{8} \sum_{j=0}^{\infty}(2 j+1) \Delta_{Q j}^{\mathrm{s}}(k) M_{(j) a b}(\hat{\mathbf{k}}, \hat{\mathbf{n}})
\end{aligned}
$$

where the second line defines the tensor $M_{(j) a b}$; i.e., the tensor $M_{(j)}^{a b}$ (with raised indices) takes the form

$$
M_{(j)}^{a b}(\hat{\mathbf{k}}=\hat{\mathbf{z}}, \hat{\mathbf{n}})=P_{j}(\cos \theta)\left(\begin{array}{cc}
1 & 0 \\
0 & -\csc ^{2} \theta
\end{array}\right)
$$

This $j$ expansion is not an expansion in tensor spherical harmonics and, as we shall see, the harmonic content of the $M_{(j)}^{a b}$ tensor, while peaked around $l=j$, has significant contributions from $l$ far from $j$.

We now proceed to re-express the above representation of polarization in terms of tensor spherical harmonics. The $\mathrm{G}$ multipole coefficients of the pattern in Eq. (4.6), given by Eq. (2.17), are

$$
\begin{aligned}
a_{(l m)}^{\mathrm{G}, \mathrm{s}}(\mathbf{k}) & =N_{l} \int d \hat{\mathbf{n}} Y_{(l m)}^{*}(\hat{\mathbf{n}}) \mathcal{P}^{a b}: a b \\
& =\frac{N_{l}}{8} \sum_{j=0}^{\infty}(2 j+\hat{\mathbf{n}}) \\
&
\end{aligned}
$$

We may compute $M_{j}^{a b}: a b$ in spherical polar coordinates by substituting Eq. (4.7) into Eq. (A13), obtaining

$$
M_{(j): a b}^{a b}=\left(1-x^{2}\right) P_{j}^{\prime \prime}(x)-4 x P_{j}^{\prime}(x)-2 P_{j}(x), \quad x \equiv \hat{\mathbf{k}} \cdot \hat{\mathbf{n}} .
$$

This can be simplified using the definition of the associated Legendre polynomials $P_{l}^{m}(x)$ and the recursion relation Eq. (B5), giving

$$
M_{(j): a b}^{a b}=-P_{j}^{2}(x)-2\left(j^{2}+j+1\right) P_{j}(x) .
$$


$P_{j}^{2}(x)$ can be represented as a finite series in $P_{l}(x)$ using the integral Eq. (B10), giving finally

$$
\begin{gathered}
M_{(j): a b}^{a b}=-\sum_{l=0}^{j}(l+1)(l+2) c_{l j} P_{l}(\hat{\mathbf{k}} \cdot \hat{\mathbf{n}}), \\
c_{l j} \equiv \begin{cases}1 & l=j, \\
\frac{2(2 l+1)}{(l+1)(l+2)} & l-j \text { even and } l<j, \\
0, & l-j \text { odd or } l>j .\end{cases}
\end{gathered}
$$

Making use of Eq. (B12) and orthonormality of the spherical harmonics,

$$
\begin{aligned}
\int d \hat{\mathbf{n}} Y_{(l m)}^{*}(\hat{\mathbf{n}}) M_{(j): a b}^{a b}(\hat{\mathbf{n}})= & -(l+1)(l+2) c_{l j} \\
& \times \sqrt{\frac{4 \pi}{2 l+1}} \delta_{m 0}
\end{aligned}
$$

and

$$
a_{(l m)}^{\mathrm{G}, \mathrm{s}}(\mathbf{k})=\frac{1}{4} \sqrt{\pi(2 l+1)} \delta_{m 0} \Delta_{\mathrm{G} l}^{\mathrm{s}}(k)
$$

where

$$
\Delta_{\mathrm{G} l}^{\mathrm{s}}(k) \equiv-\left(\frac{2(l+1)(l+2)}{l(l-1)}\right)^{1 / 2} \sum_{j=l}^{\infty}\left(\frac{2 j+1}{2 l+1}\right) c_{l j} \Delta_{Q j}^{\mathrm{s}}(k) .
$$

This infinite sum is, as shown below, equivalent to the finite sum of Eq. (4.24). The contribution to $C_{l}^{\mathrm{G}}$ from this k mode is

$$
C_{l}^{\mathrm{G}, \mathrm{s}}(\mathbf{k})=\frac{1}{2 l+1} \sum_{m=-l}^{l}\left|a_{(l m)}^{\mathrm{G}, \mathrm{s}}(\mathbf{k})\right|^{2}=\frac{\pi}{16}\left|\Delta_{\mathrm{G} l}^{\mathrm{s} l}(k)\right|^{2} .
$$

The moments $C_{l}$ are rotationally invariant, so assuming statistical isotropy, which guarantees that the different $\mathbf{k}$ modes are uncorrelated, the total contribution of all scalar modes to $C_{l}^{\mathrm{G}}$ is

$$
C_{l}^{\mathrm{G}, \mathrm{scalar}}=\int \frac{d^{3} \mathbf{k}}{(2 \pi)^{3}} C_{l}^{\mathrm{G}, \mathrm{s}}(\mathbf{k})=\frac{1}{32 \pi} \int k^{2} d k\left|\Delta_{\mathrm{G} l}^{\mathrm{s}}(k)\right|^{2} .
$$

The calculation of $C_{l}^{\mathrm{C}, \mathrm{s}}$ is similar with the replacements $\mathcal{P}^{a b}: a b \rightarrow \mathcal{P}^{a b}{ }_{a c} \epsilon_{b}^{c}$ and $M_{(j): a b}^{a b} \rightarrow M_{(j): a c}^{a b} \epsilon_{b}^{c}$ in Eq. (4.8) [cf., Eqs. (2.17) and (2.18)]. However, substituting Eq. (4.7) into Eq. (A14) yields $M_{(j): a c}^{a b} \epsilon_{b}^{c}=0$ since $M_{(j)}^{a b}$ is diagonal and independent of $\phi$. This is just what we expect: Since $M_{(j)}^{a b}$ is even under parity while $\epsilon_{b}^{c}$ is odd, the product must integrate to zero. Thus for scalar perturbations, $a^{\mathrm{C}, \mathrm{s}}(\mathbf{k})_{(l m)}=0$ and

$$
C_{l}^{\mathrm{C}, \text { scalar }}=0
$$

as argued above.

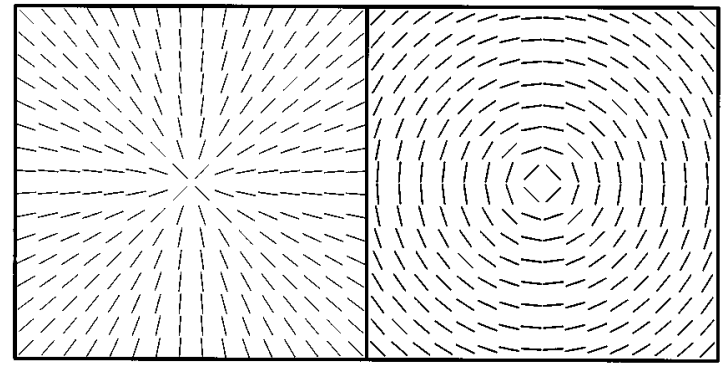

FIG. 1. The basis tensors $M_{(j)}^{a b}(k)$ traditionally used for polarization are discontinuous in the orientation of the polarization in the directions on the sky both parallel and antiparallel to $\mathbf{k}$. The discontinuity is either as depicted in the left panel or as depicted in the right panel. Note that we have just switched the sign of the polarization between the two panels.

\section{Polarization in the $\pm \hat{\mathbf{k}}$ directions}

As seen from Eq. (4.11), when $l$ is large the coefficients $c_{l j}$ at $l=j$ are much larger than "nearby" coefficients, say when $l=j-2$. If the $l=j$ term dominates, then in the smallangle approximation, i.e., for $l \gg 1$, the approximation $\Delta_{\mathrm{G} l}^{\mathrm{s}}(k) \approx \sqrt{2} \Delta_{Q l}^{\mathrm{s}}(k)$ is valid. However, this is a priori not a very good approximation since the contribution from the terms with $l+2 \leqslant j \leqslant 2 l$ to the sum of Eq. (4.14) comes to nearly as much as the contribution from the $j=l$ term (although some cancelation may result from sign changes in $\left.\Delta_{Q l}^{\mathrm{s}}\right)$. The explanation is that it takes the sum of a large number $M_{(j)}^{a b}(\hat{\mathbf{n}})$ to represent $Y_{(l 0)}^{\mathrm{G} a b}(\hat{\mathbf{n}})$. This behavior is expected for the simple reason that while the $Y_{(l m)}^{\mathrm{G} a b}$, s are smooth functions, the $M_{(j)}^{a b}$, s are not: for $\hat{\mathbf{n}}= \pm \hat{\mathbf{k}}$, i.e., when $\theta=0$ and $\pi$ (which are singular points of the spherical polar coordinate system), $M_{(j)}^{a b}$ does not go to zero [since $P_{j}(1)$ $\neq 0$ and $\left.P_{j}(-1) \neq 0\right]$. Instead the amplitude of the polarization approaches a constant but its direction varies discontinuously as illustrated in Fig. 1. To represent this discontinuous behavior as a superposition of smooth functions requires a large number of terms. In fact, the only reason why the sum of Eq. (4.11) does not contain an infinite number of terms is because it includes either $l=0$ if $j$ is even or $l=1$ if $j$ is odd, neither of which are part of the basis of harmonic STF tensors.

While the $M_{(j)}^{a b}$ basis functions are discontinuous, the physical polarization pattern must be continuous. The polarization will in fact be zero in the directions $\hat{\mathbf{n}}= \pm \hat{\mathbf{k}}$ as can be seen directly from the form of the Boltzmann equations [13]. Thus the solution of the Boltzmann equation will obey

$$
\begin{gathered}
\mathcal{P}_{a b}(\mathbf{k},+\hat{\mathbf{k}}) \propto \sum_{j=0}^{\infty}(2 j+1) \Delta_{Q j}^{\mathrm{s}}(k)=0, \\
\mathcal{P}_{a b}(\mathbf{k},-\hat{\mathbf{k}}) \propto \sum_{j=0}^{\infty}(-1)^{j}(2 j+1) \Delta_{Q j}^{\mathrm{s}}(k)=0,
\end{gathered}
$$

which in turn implies

$$
\sum_{j \geqslant 0}^{\text {even }}(2 j+1) \Delta_{Q j}^{\mathrm{s}}(k)=\sum_{j \geqslant 1}^{\text {odd }}(2 j+1) \Delta_{Q j}^{\mathrm{s}}(k)=0 .
$$


Assuming a continuous polarization pattern and substituting Eqs. (4.6) and (4.11) into Eq. (4.12) gives

$$
\begin{aligned}
& \int d \hat{\mathbf{n}} Y_{(00)}^{*}(\hat{\mathbf{n}}) \mathcal{P}^{a b}: a b(\mathbf{k}, \hat{\mathbf{n}}) \propto \sum_{j=0}^{\infty}(2 j+1) c_{0 j} \Delta_{Q j}^{\mathrm{s}}(k) \\
& =\sum_{j \geqslant 0}^{\text {even }}(2 j+1) \Delta_{Q j}^{\mathrm{s}}(k)=0, \\
& \int d \hat{\mathbf{n}} Y_{(10)}^{*}(\hat{\mathbf{n}}) \mathcal{P}^{a b}: a b(\mathbf{k}, \hat{\mathbf{n}}) \propto \sum_{j=0}^{\infty}(2 j+1) c_{1 j} \Delta_{Q j}^{\mathrm{s}}(k) \\
& =\sum_{j \geqslant 1}^{\text {odd }}(2 j+1) \Delta_{Q j}^{\mathrm{s}}(k)=0 .
\end{aligned}
$$

Thus the decomposition of $\mathcal{P}^{a b}: a b$ actually has no $l=0$ or $l=1$ content.

Define the coefficients

$$
b_{l j} \equiv \begin{cases}-\frac{l^{2}-1}{(l+1)(l+2)}, & l=j, \\ \frac{2(2 l+1)}{(l+1)(l+2)}, & l-j \text { even and } 0<j<l \\ 0, & l-j \text { odd or } j>l\end{cases}
$$

which have the property

$$
\begin{gathered}
\frac{(l+1)(l+2)}{2(2 l+1)}\left(\sum_{j=0}^{l} b_{l j}(2 j+1) \Delta_{Q j}^{\mathrm{s}}(k)\right. \\
\left.+\sum_{j=l}^{\infty} c_{l j}(2 j+1) \Delta_{Q j}^{\mathrm{s}}(k)\right) \\
= \begin{cases}\sum_{j \geqslant 0}^{\text {even }}(2 j+1) \Delta_{Q j}^{\mathrm{s}}(k), & l \text { even }, \\
\sum_{j \geqslant 1}^{\text {odd }}(2 j+1) \Delta_{Q j}^{\mathrm{s}}(k), & l \text { odd. }\end{cases}
\end{gathered}
$$

Since these sums are zero for smooth (cosmological) polarization patterns, we may use the equality

$$
\sum_{j=l}^{\infty} c_{l j}(2 j+1) \Delta_{Q j}^{\mathrm{s}}(k)=-\sum_{j=0}^{l} b_{l j}(2 j+1) \Delta_{Q j}^{\mathrm{s}}(k)
$$

in Eq. (4.14) to obtain

$$
\Delta_{\mathrm{G} l}^{\mathrm{s}}(k)=\left(\frac{2(l+1)(l+2)}{l(l-1)}\right)^{1 / 2} \sum_{j=0}^{l}\left(\frac{2 j+1}{2 l+1}\right) b_{l j} \Delta_{Q j}^{\mathrm{s}}(k) .
$$

We have transformed an infinite sum into a finite sum. While these finite sums are still somewhat cumbersome, they are significantly less complicated than previous expressions for moments of Stokes parameters obtained in the small-angle limit $[13,14]$.

The fact that continuity demands zero polarization at $\hat{\mathbf{n}}= \pm \hat{\mathbf{k}}$ is reflected in that both $Y_{(l 0) a b}^{\mathrm{G}}$ and $Y_{(l 0) a b}^{\mathrm{C}}$ are zero at $\theta=0$ and $\pi$, or equivalently that $G_{(l 0)}^{ \pm}( \pm 1)=0$; continuity in fact requires $G_{(l m)}^{ \pm}( \pm 1)=0$ for all $m$ except when $m= \pm 2$. Since the $m= \pm 2$ terms correspond to tensor perturbations when $\hat{\mathbf{k}}$ and $\hat{\mathbf{z}}$ are aligned, the polarization from tensor perturbations does not give large sums, as evidenced below. However, a similar treatment of vector perturbations ( $m= \pm 1$ ) leads to the same sort of infinite sums encountered for scalar perturbations.

\section{Cross moments}

From Eqs. (4.2) and (4.13), the contribution to the TG cross moments from a single scalar mode in the $\hat{\mathbf{k}}=\hat{\mathbf{z}}$ direction is

$$
C_{l}^{\mathrm{TG}, \mathrm{s}}(\mathbf{k})=\frac{1}{2 l+1} \sum_{m=-l}^{l}\left(a_{(l m)}^{\mathrm{T}, \mathrm{s}}(\mathbf{k})\right)^{*} a_{(l m)}^{\mathrm{G}, \mathrm{s}}(\mathbf{k})
$$

so integrating over all $\hat{\mathbf{k}}$ gives

$$
\begin{aligned}
C_{l}^{\mathrm{TG}, \mathrm{scalar}} & =\int \frac{d^{3} \mathbf{k}}{(2 \pi)^{3}} C_{l}^{\mathrm{TG}, \mathrm{s}}(\mathbf{k}) \\
& =\frac{1}{16 \pi} \int k^{2} d k \Delta_{\mathrm{G} l}^{\mathrm{s}}(k) \Delta_{I l}^{\mathrm{s}}(k) .
\end{aligned}
$$

The vanishing of $a_{(l m)}^{\mathrm{C}, \mathrm{s}}(\mathbf{k})$ also demonstrates explicitly that the moments $C_{l}^{\mathrm{TC}, \mathrm{s}}=C_{l}^{\mathrm{GC}, \mathrm{s}}=0$, as argued above.

\section{B. Tensor metric perturbations}

\section{Temperature moments}

The calculation of tensor moments proceeds in an analogous fashion. Recall, however, that tensor perturbations have two polarization states, + and $\times$. Consider a single Fourier mode with + polarization and as before choose a coordinate system with $\hat{\mathbf{z}}$ in the $\hat{\mathbf{k}}$ direction. From Eq. (7.1) in Ref. [13], the contribution of this $\mathbf{k}$ mode to the temperature anisotropy is 


$$
T(\mathbf{k}, \hat{\mathbf{n}})_{+}=\frac{T_{0}}{4} \sum_{j}(2 j+1) P_{j}(\hat{\mathbf{k}} \cdot \hat{\mathbf{n}}) \sin ^{2} \theta \cos 2 \phi \widetilde{\Delta}_{I l}^{+}(k)
$$

(note that the choice of the zero of $\phi$ is arbitrary and inconsequential), and $\widetilde{\Delta}_{I l}^{+}(k)$ is the perturbation to the photon brightness induced by this tensor mode after the Polnarev change of variables [36]. For the $\times$ polarization state, simply replace $\cos 2 \phi$ with $\sin 2 \phi$ and $\widetilde{\Delta}_{I l}^{+}$with $\widetilde{\Delta}_{I l}^{\times}$. Again, we expand this anisotropy pattern in spherical harmonics,

$$
\begin{aligned}
a_{(l m)}^{\mathrm{T},+}(\mathbf{k})= & \frac{1}{4} \sum_{j}(2 j+1) \widetilde{\Delta}_{I j}^{+}(k) \int d \hat{\mathbf{n}} P_{j}(\hat{\mathbf{k}} \cdot \hat{\mathbf{n}}) \\
& \times Y_{(l m)}^{*}(\hat{\mathbf{n}}) \sin ^{2} \theta \cos 2 \phi .
\end{aligned}
$$

Note that for $\hat{\mathbf{k}}=\hat{\mathbf{z}}, P_{j}(\cos \theta)=[4 \pi /(2 l+1)]^{1 / 2} Y_{(j 0)}$. Then Eq. (B15) can be used to express the integrand in Eq. (4.28) as a sum of products of two spherical harmonics. Orthonormality of spherical harmonics then gives

$$
\begin{aligned}
a_{(l m)}^{\mathrm{T},+}(\mathbf{k})= & \frac{1}{8}\left(\delta_{m, 2}+\delta_{m,-2}\right) \sum_{j}(2 j+1) \sqrt{\frac{4 \pi}{2 j+1}} \Delta_{I j}^{+}(k) \\
& \times\left\{\left[\frac{(j+1)(j+2)(j+3)(j+4)}{(2 j+1)(2 j+3)^{2}(2 j+5)}\right]^{1 / 2} \delta_{l, j+2}\right. \\
& +2 \sqrt{\frac{j(j+1)(j-1)(j+2)}{(2 j-1)(2 j+3)}} \delta_{l j} \\
& \left.+\left[\frac{j(j-1)(j-2)(j-3)}{(2 j-1)^{2}(2 j+1)(2 j-3)}\right]^{1 / 2} \delta_{l, j-2}\right\} .
\end{aligned}
$$

The $\delta_{l x}$ 's project out only three terms in the sum, and this reduces to

$$
\begin{aligned}
a_{(l m)}^{\mathrm{T},+}(\mathbf{k})= & \frac{1}{4} \sqrt{\pi(2 l+1)}\left(\delta_{m 2}+\delta_{m,-2}\right) \sqrt{\frac{(l+2) !}{(l-2) !}} \\
& \times\left[\frac{\Delta_{I, l-2}^{+}(k)}{(2 l-1)(2 l+1)}-\frac{2 \Delta_{I l}^{+}(k)}{(2 l+3)(2 l-1)}\right. \\
& \left.+\frac{\Delta_{I, l+2}(k)}{(2 l+3)(2 l+1)}\right] .
\end{aligned}
$$

For the $\times$ polarization state, simply replace $\left(\delta_{m 2}+\delta_{m,-2}\right)$ with $-i\left(\delta_{m 2}-\delta_{m,-2}\right)$ and $\Delta^{+}$with $\Delta^{\times}$.

The contribution of this $\mathbf{k}$ mode to $C_{l}^{\mathrm{T}}$ is then

$$
\begin{aligned}
C_{l}^{\mathrm{T},+}(\mathbf{k})= & \frac{1}{2 l+1} \sum_{m}\left|a_{(l m)}^{\mathrm{T},+}(\mathbf{k})\right|^{2} \\
= & \frac{\pi}{8} \frac{(l+2) !}{(l-2) !}\left[\frac{\Delta_{I, l-2}^{+}(k)}{(2 l-1)(2 l+1)}-\frac{2 \Delta_{I l}^{+}(k)}{(2 l+3)(2 l-1)}\right. \\
& \left.+\frac{\Delta_{I, l+2}(k)}{(2 l+3)(2 l+1)}\right]^{2},
\end{aligned}
$$

and the result for the $\times$ polarization state is the same (with the replacement $+\rightarrow \times$, of course). If the spectrum of + and $X$ states is the same (which is demanded by statistical isotropy), then the total contribution of tensor modes to the temperature anisotropy is

$$
\begin{aligned}
C_{l}^{\mathrm{T}, \text { tensor }}= & 2 \int \frac{d^{3} \mathbf{k}}{(2 \pi)^{3}} C_{l}^{\mathrm{T},+}(\mathbf{k}) \\
= & \frac{1}{8 \pi} \frac{(l+2) !}{(l-2) !} \int k^{2} d k\left[\frac{\Delta_{I, l-2}^{+}(k)}{(2 l-1)(2 l+1)}\right. \\
& \left.-\frac{2 \Delta_{I l}^{+}(k)}{(2 l+3)(2 l-1)}+\frac{\Delta_{I, l+2}(k)}{(2 l+3)(2 l+1)}\right]^{2},
\end{aligned}
$$

which agrees with the results of previous calculations $[13,12,37]$.

\section{Polarization moments}

Finally, we calculate the polarization moments from tensor perturbations. The Stokes parameters induced by a single tensor Fourier mode with + polarization in the direction $\hat{\mathbf{n}}=(\theta, \phi)$ are [13]

$$
\begin{aligned}
& Q(\mathbf{k}, \hat{\mathbf{n}})_{+}= \frac{T_{0}}{4} \sum_{j}(2 j+1) P_{j}(\cos \theta)\left(1+\cos ^{2} \theta\right) \\
& \times \cos 2 \phi \widetilde{\Delta}_{Q j}^{+}(k), \\
& U(\mathbf{k}, \hat{\mathbf{n}})_{+}=\frac{T_{0}}{4} \sum_{j}(2 j+1) P_{j}(\cos \theta) 2 \cos \theta \sin 2 \phi \widetilde{\Delta}_{Q j}^{+}(k),
\end{aligned}
$$

where again $\widetilde{\Delta_{Q l}^{\mathrm{s}}}(k)$ are Legendre coefficients of the photon polarization brightness for tensor metric perturbations with + polarization. Note $U$ is the opposite sign from Ref. [13] because the coordinate system there has opposite orientation from the one here. The polarization tensor is thus

$$
\mathcal{P}_{+}^{a b}(\mathbf{k}, \hat{\mathbf{n}})=\frac{T_{0}}{8} \sum_{j}(2 j+1) \Delta_{Q j}^{+}(k) M_{j}^{a b}(\hat{\mathbf{n}}),
$$

with

$$
M_{(j)}^{a b}(\hat{\mathbf{n}})=P_{j}(\cos \theta)\left(\begin{array}{cc}
\left(1+\cos ^{2} \theta\right) \cos 2 \phi & -2 \cot \theta \sin 2 \phi \\
-2 \cot \theta \sin 2 \phi & -\left(1+\cos ^{2} \theta\right) \csc ^{2} \theta \cos 2 \phi
\end{array}\right) .
$$


For $\times$ polarization, make the replacements $\cos 2 \phi \rightarrow \sin 2 \phi$ and $\sin 2 \phi \rightarrow-\cos 2 \phi$.

Now we calculate the multipole moments induced by this single tensor mode. From Eq. (2.17) above,

$$
\begin{aligned}
a_{(l m)}^{\mathrm{G},+}(\mathbf{k})= & \frac{N_{l}}{8} \sum_{j}(2 j+1) \widetilde{\Delta}_{Q j}^{+}(k) \\
& \times \int d \hat{\mathbf{n}} Y_{(l m)}^{*}(\hat{\mathbf{n}}) M_{(j): a b}^{a b}(\hat{\mathbf{n}}) .
\end{aligned}
$$

Calculation of this $M_{(j): a b}^{a b}$ is straightforward using Eq. (A13), but the algebra is considerably longer than the scalar case. The result is

$$
\begin{aligned}
M_{(j): a b}^{a b}= & \cos 2 \phi\left[12\left(1-x^{2}\right) P_{j}(x)+8 x\left(1-x^{2}\right) P_{j}^{\prime}(x)\right. \\
& \left.+\left(1-x^{4}\right) P_{j}^{\prime \prime}(x)\right],
\end{aligned}
$$

with $x=\cos \theta$. The $\times$ perturbations give the same result with $\cos 2 \phi \rightarrow \sin 2 \phi$. Because of this azimuthal dependence, the integral in Eq. (4.37) is nonzero only for $m= \pm 2$. Using Eqs. (B1), (B6), and (B16), we get

$$
\begin{aligned}
M_{(j): a b}^{a b}= & \cos 2 \phi\left[\frac{(j+3)(j+4) P_{j+2}^{2}}{(2 j+1)(2 j+3)}+\frac{6 j(j+1) P_{j}^{2}}{(2 j+3)(2 j-1)}\right. \\
& \left.+\frac{(j-2)(j-3) P_{j-2}^{2}}{(2 j+1)(2 j-1)}\right] .
\end{aligned}
$$

This is just a sum of the three spherical harmonics with $m=2$ and the three with $m=-2$. The integral in Eq. (4.37) is

$$
\begin{aligned}
\int d \hat{\mathbf{n}} Y_{(l m)}(\hat{\mathbf{n}}) M_{(j): a b}^{a b}(\hat{\mathbf{n}})= & \sqrt{\frac{\pi}{2 l+1} \frac{(l+2) !}{(l-2) !}}\left(\delta_{m 2}+\delta_{m,-2}\right) \\
& \times\left[\frac{(l+1)(l+2) \delta_{l, j+2}}{(2 l-3)(2 l-1)}\right. \\
& +\frac{6 l(l+1) \delta_{l j}}{(2 l+3)(2 l-1)} \\
& \left.+\frac{l(l-1) \delta_{l, j-2}}{(2 l+5)(2 l+3)}\right],
\end{aligned}
$$

which results in

$$
\begin{aligned}
a_{(l m)}^{\mathrm{G},+}(\mathbf{k})= & \frac{1}{8}\left(\delta_{m 2}+\delta_{m,-2}\right) \sqrt{2 \pi(2 l+1)} \\
& \times\left[\frac{(l+2)(l+1) \Delta_{Q, l-2}^{+}(k)}{(2 l-1)(2 l+1)}+\frac{6 l(l+1) \Delta_{Q l}^{+}(k)}{(2 l+3)(2 l-1)}\right. \\
& \left.+\frac{l(l-1) \Delta_{Q, l+2}^{+}(k)}{(2 l+1)(2 l+3)}\right] .
\end{aligned}
$$

As before, each $\mathbf{k}$ mode and each polarization state contributes to $C_{l}$ in the same way, so integrating over $\mathbf{k}$ and multiplying by two (for the two polarization states) gives

$$
\begin{aligned}
C_{l}^{\mathrm{G}, \text { tensor }}= & \frac{1}{16 \pi} \int k^{2} d k\left[\frac{(l+2)(l+1) \Delta_{Q, l-2}^{+}(k)}{(2 l-1)(2 l+1)}\right. \\
& \left.+\frac{6 l(l+1) \Delta_{Q l}^{+}(k)}{(2 l+3)(2 l-1)}+\frac{l(l-1) \Delta_{Q, l+2}^{+}(k)}{(2 l+1)(2 l+3)}\right]^{2} .
\end{aligned}
$$

Therefore, $C_{l}^{\mathrm{G} \text {,tensor }}$ is due to three Legendre expansion coefficients $\Delta_{Q l}^{+}(k)$ as opposed to an infinite sum as in the scalar case.

The derivation of the multipole moments for the $\mathrm{C}$ harmonics is similar. From Eq. (2.18) above,

$$
\begin{aligned}
a_{(l m)}^{\mathrm{C}+}(\mathbf{k})= & \frac{N_{l}}{8} \sum_{j}(2 j+1) \widetilde{\Delta}_{Q j}^{+}(k) \\
& \times \int d \hat{\mathbf{n}} Y_{(l m)}^{*}(\hat{\mathbf{n}}) M_{(j): a c}^{a b}(\hat{\mathbf{n}}) \boldsymbol{\epsilon}^{c}{ }_{b} .
\end{aligned}
$$

This time we get for the + gravity wave polarization

$$
\begin{aligned}
M_{(j): a c}^{a b} \epsilon_{b}^{c}= & \sin 2 \phi\left[-8\left(1-x^{2}\right) P_{j}^{\prime}(x)-2 x\left(1-x^{2}\right) P_{j}^{\prime \prime}(x)\right] \\
= & \sin 2 \phi\left[-\frac{2(j+3)}{2 j+1} P_{j+1}^{2}(x)\right. \\
& \left.-\frac{2(j-2)}{2 j+1} P_{j-1}^{2}(x)\right]
\end{aligned}
$$

where the second equality uses the identities (B6) and (B7). For $\times$ polarization states, replace $\sin 2 \phi \rightarrow-\cos 2 \phi$. The multipole coefficients are then

$$
\begin{aligned}
a^{\mathrm{C},+}(\mathbf{k})_{l m}= & -\frac{i}{4}\left(\delta_{m 2}-\delta_{m-2}\right) \sqrt{\frac{2 \pi}{2 l+1}}\left[(l+2) \widetilde{\Delta}_{Q l-1}^{+}(k)\right. \\
& \left.+(l-1) \widetilde{\Delta}_{Q l+1}^{+}(k)\right] .
\end{aligned}
$$

The $X$ perturbations give the same result except for a minus sign between the Kronecker $\delta$ 's and an overall factor of $i$. Again assuming equal contributions from both + and $\times$ tensor perturbations,

$$
C_{l}^{\mathrm{C}, \text { tensor }}=\frac{1}{4 \pi} \int d k k^{2}\left[\frac{l+2}{2 l+1} \widetilde{\Delta}_{Q l-1}^{+}(k)+\frac{l-1}{2 l+1} \widetilde{\Delta}_{Q l-1}^{+}(k)\right]^{2} .
$$

This calculation verifies our qualitative arguments that tensor modes will produce a $\mathrm{C}$ polarization field. 


\section{Cross moments}

From Eqs. (4.30) and (4.41), the nonzero cross moments are

$$
\begin{aligned}
C_{l}^{\mathrm{TG}, \text { tensor }}= & \frac{1}{8 \pi N_{l}} \int k^{2} d k\left[\frac{\widetilde{\Delta}_{I, l-2}^{+}(k)}{(2 l-1)(2 l+1)}\right. \\
& \left.-\frac{2 \widetilde{\Delta}_{I l}^{+}(k)}{(2 l+3)(2 l-1)}+\frac{\widetilde{\Delta}_{I, l+2}^{+}(k)}{(2 l+3)(2 l+1)}\right] \\
& \times\left[\frac{(l+2)(l+1) \widetilde{\Delta}_{Q, l-2}^{+}(k)}{(2 l-1)(2 l+1)}+\frac{6 l(l+1) \widetilde{\Delta}_{Q l}^{+}(k)}{(2 l+3)(2 l-1)}\right. \\
& \left.+\frac{l(l-1) \widetilde{\Delta}_{Q, l+2}^{+}(k)}{(2 l+1)(2 l+3)}\right],
\end{aligned}
$$

assuming equal contributions from the + and $\times$ tensor perturbations. Note the $N_{l}^{-1}$ in the prefactor comes from the temperature coefficient, not from the polarization coefficient.

Both the temperature and $\mathrm{G}$ multipole coefficients for a $\mathbf{k}$ tensor mode with + polarization $a_{(l m)}^{\mathrm{T},+}(\mathbf{k})$ and $a_{(l m)}^{\mathrm{G},+}(\mathbf{k})$ are proportional to $\left(\delta_{m 2}+\delta_{m,-2}\right)$ [and similarly for $\times$ modes with the sum replaced by a difference. On the other hand, the corresponding $\mathrm{C}$ multipole coefficients $a_{(l m)}^{\mathrm{C},+}(\mathbf{k})$ are proportional to $\left(\delta_{m 2}-\delta_{m,-2}\right)$ (and the same with the difference replaced by a sum for $\times$ modes)]. Therefore, after summing over $m$, the cross moments $C_{l}^{\mathrm{TC} \text {,tensor }}$ and $C_{l}^{\mathrm{GC} \text {, tensor }}$ for tensor perturbations vanish. This is a consequence of the symmetry arguments mentioned above.

\section{Summary}

In this section we have calculated the CMB temperature and polarization multipole moments for both scalar and tensor perturbations. For theories in which the perturbations have a Gaussian distribution (such as inflationary models), this set of multipole moments fully specifies the statistical properties of the combined temperature or polarization map. In virtually all theories considered, scalar and tensor perturbations are statistically independent, so their contributions to the CMB temperature anisotropy and polarization add in quadrature. Even if they are not statistically independent, angular orthogonality of the different modes (i.e., scalar, vector, and tensor modes in the $\hat{\mathbf{z}}$ direction induce nonzero multipole coefficients only for $m=0, m= \pm$, and $m= \pm 2$, respectively) guarantees that the contributions of scalar, vector, and tensor perturbations to the multipole moments will add in quadrature. Therefore, for Gaussian theories, the map will be fully described by $C_{l}^{\mathrm{T}}=C_{l}^{\mathrm{T} \text {,scalar }}+C_{l}^{\mathrm{T} \text {,tensor }}[\mathrm{cf}$. Eqs. (4.4) and (4.32)], $C_{l}^{\mathrm{G}}=C_{l}^{\mathrm{G} \text {,scalar }}+C_{l}^{\mathrm{G} \text {,tensor }}$ [cf. Eqs. (4.16) and (4.42)], $\quad C_{l}^{\mathrm{C}}=C_{l}^{\mathrm{C} \text {,tensor }} \quad[\mathrm{cf} . \quad$ Eqs. (4.46)], and $C_{l}^{\mathrm{TG}}=C_{l}^{\mathrm{TG} \text {,scalar }}+C_{l}^{\mathrm{TG} \text {,tensor }}[$ cf. Eqs. (4.26) and (4.47)]. For non-Gaussian theories, $n$-point correlation functions with $n>2$ may be nonzero.

Equation (4.4) for the temperature moments from scalar perturbations is written as an integral of a square of a single $\Delta_{I l}$. However, all of the other moments are written as squares of a sum of several $\Delta_{I l}$ 's and/or $\Delta_{Q l}$ 's. This is because a spherical-harmonic decomposition is not natural for a Stokes parameter $Q$, which is a tensor component, nor for the brightness perturbation from tensor modes. The calculation can be reformulated using a tensor-harmonic expansion for these quantities, which provide a natural basis [31].

\section{Line-of-sight approach}

A very efficient and accurate algorithm for computation of multipole moments has recently been given by Seljak and Zaldarriaga [38] and applied to the polarization multipole moments [21] (hereafter, SZ). Although significantly different in appearance, their spin-harmonic formalism is equivalent to our tensor-harmonic formalism. Here, we briefly compare our results with theirs. Although the formalisms differ and the calculations are somewhat lengthy, we find that the results agree, which gives us confidence in both sets of results. Furthermore, by identifying the moments in their paper with those in ours, their numerical code (which has been made publicly available) can be used to compute the multipole moments presented in this paper.

Consider, for example, the $\mathrm{G}$ polarization moments from scalar perturbations. According to Eqs. (12) and (14) in SZ, the polarization pattern induced on the sky by a scalar $\mathbf{k}$ mode in the $\hat{\mathbf{z}}$ direction [i.e., their version of our Eq. (4.5)] is

$$
Q(\hat{\mathbf{n}})=\frac{3}{4}\left(1-\cos ^{2} \theta\right) \int_{0}^{\tau_{0}} d \tau e^{i k \tau \cos \theta} g(\tau) \Pi(k, \tau) \xi(\mathbf{k}),
$$

and $U(\hat{\mathbf{n}})=0$, where $\tau$ is the conformal time, $g(\tau)$ is a visibility function, $\Pi$ is a combination of intensity and polarization perturbations, and $\xi(\mathbf{k})$ is an amplitude for the scalar mode (see SZ for more details). Therefore, the polarization tensor induced by this scalar mode is

$P^{a b}(\hat{\mathbf{n}})=\frac{3}{4} \xi(\mathbf{k}) \int_{0}^{\tau_{0}} d \tau g(\tau) \Pi(k, \tau) e^{i k \tau \cos \theta}\left(\begin{array}{cc}1 & 0 \\ 0 & -\csc ^{2} \theta\end{array}\right)$.

Using the rules of covariant differentiation, we find that

$$
\begin{aligned}
P_{: a b}^{a b}(\hat{\mathbf{n}})= & \frac{3}{8} \xi(\mathbf{k}) \int_{0}^{\tau_{0}} d \tau g(\tau) \Pi(k, \tau) \\
& \times\left(\frac{\partial^{2}}{\partial \theta^{2}}+3 \frac{\cos \theta}{\sin \theta} \frac{\partial}{\partial \theta}-2\right) e^{i k \tau \cos \theta} \\
= & \frac{3}{8} \xi(\mathbf{k}) \int_{0}^{\tau_{0}} d \tau g(\tau) \Pi(k, \tau) \frac{d^{2}}{d(\cos \theta)^{2}} \\
& \times\left[\left(1-\cos ^{2} \theta\right)^{2} e^{i k \tau \cos \theta}\right],
\end{aligned}
$$

which agrees with their $\Delta_{\widetilde{E}}^{(S)} / 2$ given in their Eq. (15). The $C_{l}^{\mathrm{G} \text {,scalar }}$ moments are obtained by plugging this into Eq. (2.17), squaring, summing over $m$, and then integrating over k. We then find that our results agree with theirs [realizing that our $(2 \pi)^{3}$ Fourier conventions differ from theirs] if we identify $C_{l}^{\mathrm{G}}=C_{E l} / 2$, where $C_{E l}$ are their electric-type moments. We have further checked that our temperature moments agree with theirs (with no factor of two), and that our 
C moments are half their $B$ moments: $C_{l}^{\mathrm{C}}=C_{B l} / 2$. Our $C_{l}^{\mathrm{TG}}$ are equal to their $C_{C l} / \sqrt{2}$. Although we do not present it here, we have checked their tensor-mode calculations as well. Although significantly more involved, we still find agreement; for example, compare their $\mathcal{E}(x)$ and $\mathcal{B}(x)$ with our Eqs. (4.38) and (4.44). The identification of our polarization moments with theirs is also consistent with our $w_{P}^{-1}$ in Eq. (3.18) being half theirs.

\section{TWO-POINT CORRELATION FUNCTIONS}

In this section, we relate the multipole moments $C_{l}^{\mathrm{T}}$, $C_{l}^{\mathrm{G}}, C_{l}^{\mathrm{C}}$, and $C_{l}^{\mathrm{TG}}$ to two-point correlation functions of temperature and Stokes parameters. This will make contact with previous work on the subject. We also derive flat-sky limits useful for analyzing maps of small sky patches.

\section{A. Correlations between temperature and stokes parameters}

The linear-polarization state at any given point is specified completely by the Stokes parameter $Q$ and $U$, but these quantities depend on the coordinate system which one chooses. On the other hand, we know that $Q$ and $U$ transform as the components of a STF $2 \times 2$ tensor, so given $Q$ and $U$ in some coordinate system, we can always determine $Q^{\prime}$ and $U^{\prime}$ in any other coordinate system.

The Universe is assumed to be statistically isotropic, so it is possible to construct two-point correlation functions which depend only on the angular separation between the two points. But simply correlating $Q$ and $U$ in a particular coordinate system gives correlation functions which depend on the positions of the points being correlated as well the angular separation. This is what has been done in previous published work.

A coordinate-independent set of correlation functions can be expressed in terms of the ones which have appeared in the previous literature. The prescription is simply to define correlation functions of Stokes parameters with respect to axes which are parallel and perpendicular to the great arc (or geodesic) connecting the two points being correlated. So $Q_{r}$ is the difference in intensities in two linear-polarization states parallel and perpendicular to the great arc connecting the two points, and $U_{r}$ is the difference in two linear-polarization states which lie $45^{\circ}$ away from the parallel and perpendicular. The three quantities $T, Q_{r}$, and $U_{r}$ have six correlation functions between them: $\langle T T\rangle,\left\langle U_{r} U_{r}\right\rangle,\left\langle Q_{r} Q_{r}\right\rangle,\left\langle Q_{r} T\right\rangle$, $\left\langle Q_{r} U_{r}\right\rangle$, and $\left\langle U_{r} T\right\rangle$. However, only four can be nonzero. Although $Q_{r}$ and $T$ are invariant under reflection along the great arc connecting the two points being correlated, $U_{r}$ changes sign. Therefore, the expectation values $\left\langle Q_{r} U_{r}\right\rangle$ and $\left\langle U_{r} T\right\rangle$ must be zero from statistical isotropy. This is as expected: four nonzero sets of moments $C_{l}^{\mathrm{T}}, C_{l}^{\mathrm{G}}, C_{l}^{\mathrm{C}}$, and $C_{l}^{\mathrm{TG}}$ describe the map. Correspondingly, four nonzero correlation functions provide an equivalent statistical description.

We begin with the familiar $\langle T T\rangle$ correlation function,

$$
C^{\mathrm{T}}(\theta)=\left\langle\frac{T\left(\hat{\mathbf{n}}_{1}\right)}{T_{0}} \frac{T\left(\hat{\mathbf{n}}_{2}\right)}{T_{0}}\right\rangle_{\hat{\mathbf{n}}_{1} \cdot \hat{\mathbf{n}}_{2}=\cos \theta} .
$$

The correlation function depends only on the angular separation of the two points, so in calculating it, we may choose one point to be at the north pole, $(0,0)$, and the other to be on the $\phi=0$ longitude at a distance $\theta$ from the north pole, $(\theta, 0)$. Then expand $T(\hat{\mathbf{n}})$ in terms of spherical harmonics as in Eq. (2.8) and note that $Y_{(l m)}(0,0)=\delta_{m 0} \sqrt{(2 l+1) /(4 \pi)}$. So

$$
\begin{aligned}
C^{\mathrm{T}}(\theta) & =\left\langle\frac{T(0,0)}{T_{0}} \frac{T(\theta, 0)}{T_{0}}\right\rangle \\
& =\sum_{l m l^{\prime} m^{\prime}}\left\langle a_{(l m)}^{\mathrm{T} *} a_{l^{\prime} m^{\prime}}^{\mathrm{T}}\right\rangle Y_{(l m)}^{*}(0,0) Y_{\left(l^{\prime} m^{\prime}\right)}(\theta, 0) \\
& =\sum_{l m l^{\prime} m^{\prime}} C_{l}^{\mathrm{T}} \delta_{l l^{\prime}} \delta_{m m^{\prime}} \sqrt{\frac{2 l+1}{4 \pi}} \delta_{m 0} Y_{\left(l^{\prime} m^{\prime}\right)}(\theta, 0) \\
& =\sum_{l} \frac{2 l+1}{4 \pi} C_{l}^{\mathrm{T}} P_{l}(\cos \theta),
\end{aligned}
$$

where we have used Eq. (3.1) to go from the second to the third line. This recovers the well-known result for the temperature autocorrelation function.

The derivation of the polarization correlation functions will proceed analogously (and is similar to the case for weaklensing correlation functions [28]), and requires $W_{(l m)}$ and $X_{(l m)}$ at the north pole. Using the asymptotic relations

$$
\begin{gathered}
P_{l}^{m}(\cos \theta) \sim \frac{(-1)^{(m+|m|) / 2}}{2^{|m|}|m| !} \frac{(l+|m|) !}{(l-|m|) !} \theta^{|m|}, \quad \theta \rightarrow 0 \\
\quad(m \neq 0), \\
P_{l}(\cos \theta) \sim 1-\frac{1}{4} l(l+1) \theta^{2}, \quad \theta \rightarrow 0,
\end{gathered}
$$

it is straightforward to show that for $|m| \geqslant 2, X_{(l m)}$ and $Y_{(l m)}$ are both asymptotic to $\theta^{|m|-2}$ as $\theta \rightarrow 0$, so they are nonzero at $\theta=0$ only for $|m|=2$; for $m=0$ and $m=1$, they are asymptotic to $\theta^{2}$ and $\theta$. After a little algebra,

$$
W_{(l m)}(0,0)=\frac{1}{2} \sqrt{\frac{2 l+1}{4 \pi} \frac{(l+2) !}{(l-2) !}}\left(\delta_{m 2}+\delta_{m,-2}\right)
$$

and

$$
X_{(l m)}(0,0)=\frac{i}{2} \sqrt{\frac{2 l+1}{4 \pi} \frac{(l+2) !}{(l-2) !}}\left(\delta_{m 2}-\delta_{m,-2}\right) .
$$

Now consider the $\langle Q Q\rangle$ correlation function

$$
C^{\mathrm{Q}}(\theta)=\left\langle\frac{Q_{r}\left(\hat{\mathbf{n}}_{1}\right)}{T_{0}} \frac{Q_{r}\left(\hat{\mathbf{n}}_{2}\right)}{T_{0}}\right\rangle_{\hat{\mathbf{n}}_{1} \cdot \hat{\mathbf{n}}_{2}=\cos \theta},
$$

where, once again, the Stokes parameters $Q_{r}$ are defined with respect to axes parallel and perpendicular to the great arc connecting $\hat{\mathbf{n}}_{1}$ and $\hat{\mathbf{n}}_{2}$. As in the temperature case, choose one point to be at the north pole and another a distance $\theta$ away along the $\phi=0$ longitude. This choice has the added advantage that the great arc connecting these two points is along the $\theta$ direction, so we can use the $Q$ defined in the $(\hat{\theta}, \hat{\phi})$ coordinate system, Eq. (3.3). However, in this coordinate 
system, the definition of $Q$ at the north pole is, strictly speaking, ambiguous. Therefore, we always consider a point on the $\phi=0$ longitude which is infinitesimally close to the north pole; in other words, $Q(0,0)$ really means $\lim _{\theta \rightarrow 0} Q(\theta, 0)$. Using Eq. (3.3) for the Stokes parameters, the $\langle Q Q\rangle$ correlation function is then

$$
\begin{aligned}
C^{\mathrm{Q}}(\theta)= & \left\langle\frac{Q(0,0)}{T_{0}} \frac{Q(\theta, 0)}{T_{0}}\right\rangle \\
= & \sum_{l m l^{\prime} m^{\prime}} N_{l^{\prime}} N_{l^{\prime}}\left\langle\left[a_{(l m)}^{\mathrm{G}} W_{(l m)}(0,0)-a_{(l m)}^{\mathrm{C}} X_{(l m)}(0,0)\right]\right. \\
& \left.\times\left[a_{\left(l^{\prime} m^{\prime}\right)}^{\mathrm{G} *} W_{\left(l^{\prime} m^{\prime}\right)}^{*}(\theta, 0)-a_{\left(l^{\prime} m^{\prime}\right)}^{\mathrm{C}} X_{\left(l^{\prime} m^{\prime}\right)}^{*}(\theta, 0)\right]\right\rangle \\
= & \sum_{l} \sqrt{\frac{2 l+1}{8 \pi}} N_{l}\left\{C_{l}^{\mathrm{G}}\left[W_{(l 2)}^{*}(\theta, 0)+W_{(l,-2)}^{*}(\theta, 0)\right]\right. \\
& \left.+i C_{l}^{\mathrm{C}}\left[X_{(l 2)}^{*}(\theta, 0)-X_{(l,-2)}^{*}(\theta, 0)\right]\right\}
\end{aligned}
$$

where we have used Eqs. (3.1), (5.6), and (5.5), and $\left\langle a_{(l m)}^{\mathrm{G} *} a_{(l m)}^{\mathrm{C}}\right\rangle=0$. This can be simplified using $X_{(l m)}^{*}=-X_{(l,-m)}^{*}$ and $W_{(l m)}^{*}=W_{(l,-m)}^{*}$ and the definitions in Eqs. (2.24) and (2.25), giving

$$
C^{\mathrm{Q}}(\theta)=\sum_{l} \frac{2 l+1}{2 \pi} N_{l}^{2}\left[C_{l}^{\mathrm{G}} G_{(l 2)}^{+}(\cos \theta)+C_{l}^{\mathrm{C}} G_{(l 2)}^{-}(\cos \theta)\right] .
$$

For the $\langle U U\rangle$ correlation function, the derivation is similar, giving

$$
C^{\mathrm{U}}(\theta)=\sum_{l} \frac{2 l+1}{2 \pi} N_{l}^{2}\left[C_{l}^{\mathrm{C}} G_{(l 2)}^{+}(\cos \theta)+C_{l}^{\mathrm{G}} G_{(l 2)}^{-}(\cos \theta)\right] .
$$

For the $\langle T Q\rangle$ cross-correlation function,

$$
\begin{aligned}
C^{\mathrm{TQ}}(\theta) & =\left\langle\frac{T\left(\hat{\mathbf{n}}_{1}\right)}{T_{0}} \frac{Q_{r}\left(\hat{\mathbf{n}}_{2}\right)}{T_{0}}\right\rangle_{\hat{\mathbf{n}}_{1} \cdot \hat{\mathbf{n}}_{2}=\cos \theta} \\
& =\sum_{l} \frac{2 l+1}{4 \pi} N_{l} C_{l}^{\mathrm{TG}} P_{l}^{2}(\cos \theta) .
\end{aligned}
$$

Equations (5.9), (5.10), and (5.11) are exact (i.e., there is no small-angle approximation) expressions for the polarization correlation functions.

\section{B. Multipole moments from correlation functions}

Above, we derived expressions for correlation functions in terms of multipole moments, and now we perform the inverse transform and express the multipole moments in terms of correlation functions. Begin with the temperature autocorrelation function: multiply both sides of Eq. (5.2) by the Legendre polynomial $P_{l^{\prime}}(\cos \theta)$, integrate over $\cos \theta$, and use the orthogonality of Legendre polynomials to obtain

$$
C_{l}^{\mathrm{T}}=2 \pi \int_{0}^{\pi} d \theta \sin \theta P_{l}(\cos \theta) C^{\mathrm{T}}(\theta)
$$

Similarly, for the polarization-temperature moments, multiply both sides of Eq. (5.11) by the associated Legendre function $P_{l^{\prime}}^{2}$, integrate, and use Eq. (B4) to obtain

$$
C_{l}^{\mathrm{TG}}=\pi N_{l} \int_{0}^{\pi} d \theta \sin \theta P_{l}^{2}(\cos \theta) C^{\mathrm{TQ}}(\theta) .
$$

The derivation of the polarization moments from the polarization autocorrelation functions is similar. Orthonormality of the tensor harmonics implies that

$$
\begin{aligned}
& \int\left[W_{(l m)}^{*}(\hat{\mathbf{n}}) W_{\left(l^{\prime} m^{\prime}\right)}(\hat{\mathbf{n}})+X_{(l m)}^{*}(\hat{\mathbf{n}}) X_{\left(l^{\prime} m^{\prime}\right)}(\hat{\mathbf{n}})\right] d \hat{\mathbf{n}} \\
& =\frac{2}{N_{l}^{2}} \delta_{l l^{\prime}} \delta_{m m^{\prime}}
\end{aligned}
$$

and it can also be shown that

$$
\int\left[-X_{(l m)}^{*}(\hat{\mathbf{n}}) W_{\left(l^{\prime} m^{\prime}\right)}(\hat{\mathbf{n}})+W_{(l m)}^{*}(\hat{\mathbf{n}}) X_{\left(l^{\prime} m^{\prime}\right)}(\hat{\mathbf{n}})\right] d \hat{\mathbf{n}}=0
$$

To do so, note that the $\phi$ dependence of $W_{(l m)}$ and $X_{(l m)}$ is just $e^{i m \phi}$, which means that the integral is immediately zero for $m \neq m^{\prime}$. For $m=m^{\prime}$, the integral over $\cos \theta$ vanishes using the explicit forms of $G_{(l m)}^{ \pm}$[28]. From Eqs. (5.9) and (5.10),

$$
\begin{aligned}
{\left[C^{\mathrm{Q}}(\theta)+C^{\mathrm{U}}(\theta)\right] e^{2 i \phi}=} & \sum_{l} \sqrt{\frac{2 l+1}{2 \pi}} N_{l}\left(C_{l}^{\mathrm{G}}+C_{l}^{\mathrm{C}}\right)\left[W_{(l 2)}\right. \\
& \left.\times(\theta, \phi)+i X_{(l 2)}(\theta, \phi)\right], \\
{\left[C^{\mathrm{Q}}(\theta)-C^{\mathrm{U}}(\theta)\right] e^{2 i \phi}=} & \sum_{l} \sqrt{\frac{2 l+1}{2 \pi}} N_{l}\left(C_{l}^{\mathrm{G}}-C_{l}^{\mathrm{C}}\right)\left[W_{(l 2)}\right. \\
& \left.\times(\theta, \phi)-i X_{(l 2)}(\theta, \phi)\right] .
\end{aligned}
$$

Then multiply both sides of the first equation by $W_{(l 2)}^{*}-i X_{(l 2)}^{*}$ and the second by $W_{(l 2)}^{*}+i X_{(l 2)}^{*}$, integrate over all directions $\hat{\mathbf{n}}$, and apply Eqs. (5.14) and (5.15), giving

$$
\begin{aligned}
C_{l}^{\mathrm{G}}+C_{l}^{\mathrm{C}}= & \sqrt{\frac{2 \pi}{2 l+1}} \frac{N_{l}}{2} \int d \hat{\mathbf{n}}\left[C^{\mathrm{Q}}(\theta)+C^{\mathrm{U}}(\theta)\right] \\
& \times e^{2 i \phi}\left[W_{(l 2)}^{*}(\hat{\mathbf{n}})-i X_{(l 2)}^{*}(\hat{\mathbf{n}})\right], \\
C_{l}^{\mathrm{G}}-C_{l}^{\mathrm{C}}= & \sqrt{\frac{2 \pi}{2 l+1}} \frac{N_{l}}{2} \int d \hat{\mathbf{n}}\left[C^{\mathrm{Q}}(\theta)-C^{\mathrm{U}}(\theta)\right] \\
& \times e^{2 i \phi}\left[W_{(l 2)}^{*}(\hat{\mathbf{n}})+i X_{(l 2)}^{*}(\hat{\mathbf{n}})\right] .
\end{aligned}
$$

Upon summing and differencing and carrying out the integration over $\phi$, we obtain

$$
\begin{aligned}
C_{l}^{\mathrm{G}}= & \pi N_{l}^{2} \int_{0}^{\pi} d \theta \sin \theta\left[C^{\mathrm{Q}}(\theta) G_{(l 2)}^{+}(\cos \theta)\right. \\
& \left.-C^{\mathrm{U}}(\theta) G_{(l 2)}^{-}(\cos \theta)\right],
\end{aligned}
$$




$$
\begin{aligned}
C_{l}^{\mathrm{C}}= & \pi N_{l}^{2} \int_{0}^{\pi} d \theta \sin \theta\left[C^{\mathrm{U}}(\theta) G_{(l 2)}^{+}(\cos \theta)\right. \\
& \left.-C^{\mathrm{Q}}(\theta) G_{(l 2)}^{-}(\cos \theta)\right],
\end{aligned}
$$

which are the desired relations giving the polarization multipole moments in terms of the polarization autocorrelation functions. Given some measured correlation functions, evaluation of Eq. (5.21) for any $l$ will probe the existence of nonscalar modes.

\section{Correlation functions in the small-angle limit}

In order to make contact with previous work and to present estimates useful for measurements on a small patch of the sky, we now derive the small-angle limit of the above expressions which give correlation functions in terms of multipole moments (and vice versa). The correlation functions given in previous work were of Stokes parameters measured in a fixed coordinate basis, whereas ours are of Stokes parameters measured with respect to the great arc connecting the two points being correlated. However, our results can be compared with previous results by taking $\phi=0$ in their expressions. Although the expressions for correlation functions in the small-angle limit given in Refs. $[13,14]$ are quite complicated, when the small-angle limit is taken consistently in all steps, the expressions simplify greatly, as emphasized in Ref. [39] (and resemble correlation functions for ellipticities of galaxies due to weak lensing from large-scale inhomogeneities $[28,40])$.

Once again we begin with the temperature moments. A useful asymptotic relation is

$$
P_{l}(\cos \theta) \sim J_{0}(s), \quad s \equiv(2 l+1) \sin (\theta / 2) \rightarrow 0,
$$

where $J_{m}(s)$ is the Bessel function of order $m$. Substituting into Eq. (5.2), approximating the sum by an integral and taking the limit $l \gg 1$ gives

$$
C^{\mathrm{T}}(\theta) \simeq \frac{1}{2 \pi} \int^{\infty} l d l J_{0}(l \theta) C_{l}^{\mathrm{T}}
$$

for $\theta \ll 1$. For the temperature-polarization cross-correlation function, we note that

$$
P_{l}^{2}(\cos \theta) \sim 4 l^{4} J_{2}(s), \quad s \rightarrow 0
$$

which gives

$$
C^{\mathrm{TQ}}(\theta) \simeq \frac{2^{3 / 2}}{\pi} \int l^{3} d l C_{l}^{\mathrm{TG}} J_{2}(l \theta)
$$

for $\theta \ll 1$, from Eq. (5.11). [28]

For the polarization autocorrelation functions, note that

$$
G_{(l m)}^{ \pm}(\cos \theta) \sim \frac{1}{4} l^{4}\left[J_{0}(s) \pm J_{4}(s)\right], \quad s \rightarrow 0 .
$$

From Eq. (5.9) we obtain

$$
C^{\mathrm{Q}}(\theta) \simeq \frac{1}{2 \pi} \int l d l\left[\left(C_{l}^{\mathrm{G}}+C_{l}^{\mathrm{C}}\right) J_{0}(l \theta)+\left(C_{l}^{\mathrm{G}}-C_{l}^{\mathrm{C}}\right) J_{4}(l \theta)\right],
$$

and from Eq. (5.10) we obtain

$$
C^{\mathrm{U}}(\theta) \simeq \frac{1}{2 \pi} \int l d l\left[\left(C_{l}^{\mathrm{G}}+C_{l}^{\mathrm{C}}\right) J_{0}(l \theta)-\left(C_{l}^{\mathrm{G}}-C_{l}^{\mathrm{C}}\right) J_{4}(l \theta)\right],
$$

for $\theta \ll 1$.

Equations (5.23), (5.25), (5.27), and (5.28) agree with the forms in Eq. (19) in Ref. [39] for $\phi=0$ and $C_{l}^{\mathrm{C}}=0$. (Also recall that $\left\langle Q_{r} U_{r}\right\rangle=\left\langle T U_{r}\right\rangle=0$.) If $C_{l}^{\mathrm{C}}=0$, then $\left\langle Q_{r} Q_{r}+U_{r} U_{r}\right\rangle$ [which depends on an integral over $J_{0}(l \theta)$ ] and $\left\langle Q_{r} Q_{r}-U_{r} U_{r}\right\rangle$ [which depends on an integral over $\left.J_{4}(l \theta)\right]$ depend on the same set of moments $C_{l}^{\mathrm{G}}$ and are therefore not independent. However, if $C_{l}^{\mathrm{C}} \neq 0$, then these correlation functions will depend on two independent sets of moments.

We can also derive expressions for the multipole moments for $l \gg 1$ in terms of a correlation function measured at small angular separations. For example, using Eq. (5.22) to approximate Eq. (5.12) for $l \gg 1$ gives

$$
C_{l}^{\mathrm{T}} \simeq 2 \pi \int J_{0}(l \theta) C^{\mathrm{T}}(\theta) \theta d \theta
$$

and using Eq. (5.24) to approximate Eq. (5.13) gives

$$
C_{l}^{\mathrm{TG}} \simeq 4 \sqrt{2} \pi l^{2} \int J_{2}(l \theta) C^{\mathrm{TQ}}(\theta) \theta d \theta .
$$

Using Eq. (5.26), we can approximate Eq. (5.20) by

$$
\begin{aligned}
C_{l}^{\mathrm{G}} \simeq & \frac{\pi}{2} \int\left(C^{Q}(\theta)\left[J_{0}(l \theta)+J_{4}(l \theta)\right]\right. \\
& \left.-C^{U}(\theta)\left[J_{0}(l \theta)-J_{4}(l \theta)\right]\right) \theta d \theta
\end{aligned}
$$

and Eq. (5.21) by

$$
\begin{aligned}
C_{l}^{\mathrm{C}} \simeq & \frac{\pi}{2} \int\left\{C^{U}(\theta)\left[J_{0}(l \theta)+J_{4}(l \theta)\right]\right. \\
& \left.-C^{Q}(\theta)\left[J_{0}(l \theta)-J_{4}(l \theta)\right]\right\} \theta d \theta .
\end{aligned}
$$

If any nonzero $C_{l}^{\mathrm{C}}$ is found in this way with correlation functions measured on a small patch of the sky, it is an indication of vector or tensor modes.

\section{SUMMARY AND DISCUSSION}

This paper provides a detailed and complete formalism for characterizing polarization fluctuations in a full-sky map of the cosmic microwave background. We give explicit forms for tensor spherical harmonics, in which the polarization can be expanded in direct analogy to the expansion of temperature perturbations in the usual spherical harmonics. The tensor harmonics are numerically just as easy to evaluate as spherical harmonics, so polarization map simulation and analysis will be no more cumbersome than in the temperature case. 
The most important physics results presented here are that of the six sets of multipole coefficients describing the correlations in a temperature or polarization map, two must be zero if the Universe is parity invariant, and a third vanishes for scalar metric perturbations. The moments $C_{l}^{\mathrm{C}}$, which are nonzero only for vector and tensor metric perturbations, are in principle an unambiguous probe of primordial gravity waves and vorticity $[19,20]$. A cosmological contribution to the moments $C_{l}^{\mathrm{TC}}$ or $C_{l}^{\mathrm{CG}}$ would demonstrate a remarkable handedness to the primordial perturbation spectrum. A much more likely and practical use of these moments is to monitor foreground microwave emission. We also note that $\mathrm{CMB}$ polarization may give useful information on primordial magnetic fields $[17,41]$ and galaxy cluster magnetic fields [42]. Measurement of polarization in the Sunyaev-Zeldovich effect can be used to measure cluster transverse velocities [43] and/or the $\mathrm{CMB}$ quadrupole moment incident on the cluster [44].

Most current microwave background codes calculate the Legendre coefficients of the radiation brightness in Fourier space [35]. We have derived exact expressions for all of the multipole moments in terms of these brightness coefficients. For tensor metric perturbations, the expressions are particularly simple and trivial to implement numerically. The result for scalar perturbations is somewhat more complex, involving an infinite sum over the brightness moments. However, the contribution of the sum to the final expression for the multipole moments is only significant for the lowest moments, so the overall cost of the computation should only increase slightly. The formulas for the multipole moments derived in this paper should allow for relatively simple conversion of existing $\mathrm{CMB}$ codes.

Of course, a cosmological signal will have to be distinguished from foreground contamination. Synchrotron emission from our galaxy is highly polarized [45], and extragalactic radio sources may also contribute significantly [46]. The amplitude of these foreground polarization sources is unknown at the present time. Since both likely foregrounds have a spectral dependence substantially different from the blackbody CMB spectrum, the usual techniques for subtracting foregrounds from temperature maps should also work for polarization [47].

Of course, simply attaining the necessary sensitivity to make any polarization detection will be a great experimental accomplishment. The MAP satellite, currently being constructed, will have the sensitivity to make a statistical detection of polarization. The Planck satellite, now in the planning stage, should be capable of seeing polarization on a pixel-bypixel basis if it is configured to measure polarization. At this time, it is undecided whether Planck, which uses incoherent bolometer detectors in its most sensitive frequency channels, will sacrifice some temperature sensitivity to make polarized measurements. But optimistically, within a decade we may have in hand detailed temperature or polarization maps of the cosmic microwave background. How much cosmological information can be extracted from such maps is currently under study [34]. The formalism presented in this paper provides a basis for addressing such questions.

\section{ACKNOWLEDGMENTS}

We would like to thank Jerry Jungman, Lloyd Knox, and Uros Seljak for helpful conversations, and David Spergel and
Gary Hinshaw for stimulating questions. This work was supported by U.S. DOE Contract No. DEFG02-92-ER 40699, NASA NAG5-3091, and the Alfred P. Sloan Foundation at Columbia, NASA AST94-19400 at Fermilab, and the Harvard Society of Fellows. M.K. acknowledges the hospitality of the NASA/Fermilab Astrophysics Center and the CERN Theory Group.

\section{APPENDIX A: DIFFERENTIAL GEOMETRY ON THE SPHERE}

This appendix collects results from differential geometry, with particular application to the manifold $S^{2}$ (the twosphere), which are needed in definitions of and calculations with the tensor spherical harmonics on the celestial sphere. We use the notation $f_{, a} \equiv \partial f / \partial x^{a}$ to indicate a regular partial derivative and $f_{: a} \equiv \nabla_{a} f$ for a covariant derivative. We use the colon, ":', rather than the more traditional semicolon, " ‘," to distinguish derivatives on $S^{2}$ from four-dimensional derivatives in general relativity. All of our tensors are defined with respect to a coordinate basis. Note the metric tensor $g_{a b}$ commutes with covariant differentiation: $g_{a b: c}=0$. The determinant of the metric is denoted by $g \equiv\left\|g_{a b}\right\|$. Covariant derivatives of scalars, vectors, and (rank-two) tensors are

$$
\begin{gathered}
S_{: a}=S_{, a}, \quad V^{a}{ }_{: b}=V^{a}{ }_{, b}+V^{c} \Gamma_{b c}^{a}, \\
T^{a b}{ }_{: c}=T_{, c}^{a b}+T^{d b} \Gamma_{c d}^{a}+T^{a d} \Gamma_{c d}^{b},
\end{gathered}
$$

where the $\Gamma_{b c}^{a}$ are Christoffel symbols defined by

$$
\Gamma_{b c}^{a}=\frac{1}{2} g^{a d}\left(g_{d b, c}+g_{d c, b}-g_{b c, d}\right) .
$$

We have used the formula for the fourth derivatives of a scalar function

$$
\begin{gathered}
S_{a b}^{: a b}=\nabla^{2} \nabla^{2} S+R^{d b} S_{: d b}+\frac{1}{2} R^{: d} S_{: d}, \quad \nabla^{2} S \equiv S_{a}^{: a}, \\
R_{a b} \equiv R_{a c b}^{c}, \quad R \equiv R_{a}^{a},
\end{gathered}
$$

where $R_{a b c d}, R_{a b}$, and $R$ are the Riemann tensor, the Ricci tensor, and Ricci scalar, respectively. We have also used the formula of integration by parts

$$
\oint d \hat{\mathbf{n}} X^{a b} Y_{: a b}=-\oint d \hat{\mathbf{n}} X^{a b}{ }_{a} Y_{: b}=\oint d \hat{\mathbf{n}} X^{a b}{ }_{a b} Y,
$$

where $\oint$ indicates integration over a closed manifold with no boundary, and $d \hat{\mathbf{n}}$ is shorthand for $\sqrt{g} d x^{1} d x^{2}$.

On a two-dimensional manifold the Levi-Civita symbol is a traceless antisymmetric rank-two tensor given by

$$
\epsilon_{a b}=\sqrt{g}\left(\begin{array}{ll}
0 & 1 \\
-1 & 0
\end{array}\right)
$$


which has the simple properties

$$
\begin{gathered}
\epsilon_{c a} \epsilon_{b}^{c}=g_{a b}=-\epsilon_{a c} \epsilon_{b}^{c}, \quad \epsilon_{a b} \epsilon_{c d}=g_{a c} g_{b d}-g_{a d} g_{b c}, \\
\epsilon_{a b: c}=0 .
\end{gathered}
$$

A rank-two tensor is trace-free (or traceless) if, and only if, $g^{a b} T_{a b}=0$, and a rank-two tensor is symmetric if, and only if, $T_{a b}=T_{b a}$. In two dimensions the latter requirement is equivalent to $\epsilon^{a b} T_{a b}=0$. Linear polarization is described by a symmetric trace-free rank-two tensor, or STF for short.

The geometry of a two-dimensional manifold is particularly simple because it is determined solely by the Ricci scalar $R$. Some handy identities are

$$
\begin{aligned}
R_{a b c d} & =\frac{1}{2} R \epsilon_{a b} \epsilon_{c d}, \quad R_{a b}=\frac{1}{2} R g_{a b} \quad \epsilon^{a b} R_{a b c d} \\
& =R \epsilon_{c d} \quad \epsilon^{a c} R_{a b c d}=\frac{1}{2} R \epsilon_{b d} .
\end{aligned}
$$

Another useful identity is

$$
M^{a b} N^{c d} \epsilon_{a c} \epsilon_{b d}=-M^{a b} N_{a b}
$$

if

$$
g^{a b} M_{a b}=g^{a b} N_{a b}=\epsilon^{a b} M_{a b}=\epsilon^{a b} N_{a b}=0,
$$

i.e., for STF tensors only.

In this paper we are only interested in the geometry of the unit sphere $S^{2}$. Its geometry is nearly as simple as can be, since $R=2$. We exclusively use the explicit coordinate system defined by spherical polar coordinates $(\theta, \phi)$, where $\theta$ is the polar angle from a particular point on the sphere and $\phi$ labels the angle on circles which are centered on this same point. In these coordinates the metric is

$$
g_{a b}=\left(\begin{array}{cc}
1 & 0 \\
0 & \sin ^{2} \theta
\end{array}\right), \quad g \equiv\left\|g_{a b}\right\|=\sin ^{2} \theta,
$$

while the antisymmetric tensor is

$$
\epsilon_{a b}=\left(\begin{array}{cc}
0 & \sin \theta \\
-\sin \theta & 0
\end{array}\right), \quad \epsilon_{b}^{a}=\left(\begin{array}{cc}
0 & \sin \theta \\
-\csc \theta & 0
\end{array}\right) .
$$

From this metric the Christoffel symbols follow as

$$
\begin{gathered}
\Gamma_{\phi \phi}^{\theta}=-\sin \theta \cos \theta, \quad \Gamma_{\theta \phi}^{\phi}=\Gamma_{\phi \theta}^{\phi}=\cot \theta, \\
\Gamma_{\theta \theta}^{\theta}=\Gamma_{\theta \phi}^{\theta}=\Gamma_{\phi \theta}^{\theta}=\Gamma_{\theta \theta}^{\phi}=\Gamma_{\phi \phi}^{\phi}=0 .
\end{gathered}
$$

The explicit components of the second derivatives of a scalar function are

$$
\begin{gathered}
Y_{: a b}=Y_{, a b}-\Gamma_{a b}^{c} Y_{, c} ; \\
Y_{: \theta \theta}=Y_{, \theta \theta}, \\
Y_{: \theta \phi}=Y_{, \theta \phi}-\cot \theta Y_{, \phi}, \\
Y_{: \phi \phi}=Y_{, \phi \phi}+\sin \theta \cos \theta Y_{, \theta},
\end{gathered}
$$

while explicit expressions for some second derivatives of a symmetric rank-two tensor are

$$
\begin{aligned}
M_{a a b}^{a b}= & M_{, \theta \theta}^{\theta \theta}+2 M_{, \theta \phi}^{\theta \phi}+M_{, \phi \phi}^{\phi \phi}{ }_{, \phi \phi}-\sin \theta \cos \theta M_{, \theta}^{\phi \phi}{ }_{, \theta} \\
& +2 \cot \theta M^{\theta \theta}{ }_{, \theta}+4 \cot \theta M^{\theta \phi}{ }_{, \phi}+\left(1-3 \cos ^{2} \theta\right) M^{\phi \phi} \\
& -M^{\theta \theta},
\end{aligned}
$$

and

$$
\begin{aligned}
M_{: a c}^{a b} \epsilon_{b}^{c}= & \sin \theta\left(M_{, \theta \theta}^{\theta \phi}+M_{, \phi \theta}^{\phi \phi}\right)-\csc \theta\left(M_{, \theta \phi}^{\theta \theta}\right. \\
& \left.+M_{, \phi \theta}^{\phi \theta}{ }_{, \phi \phi}\right)-\cot \theta \csc \theta M^{\theta \theta}{ }_{, \phi}+5 \cos \theta M^{\theta \phi}{ }_{, \theta} \\
& +3 \cos \theta M^{\phi \phi}{ }_{, \phi}+3(\cos \theta \cot \theta-\sin \theta) M^{\theta \phi},
\end{aligned}
$$

which again only apply if $\epsilon^{a b} M_{a b}=0$.

\section{APPENDIX B: SPHERICAL HARMONIC AND LEGENDRE FUNCTION IDENTITIES}

In this appendix, we list some identities involving spherical harmonics and Legendre functions which we have used in our calculations.

The associated Legendre functions are defined by

$$
\begin{gathered}
P_{l}^{m}(x)=(-1)^{m}\left(1-x^{2}\right)^{m / 2} \frac{d^{m}}{d x^{m}} P_{l}(x), \quad \geqslant 0, \\
P_{l}^{-m}(x)=(-1)^{m} \frac{(l-m) !}{(l+m) !} P_{l}^{m}(x),
\end{gathered}
$$

in terms of Legendre polynomials,

$$
P_{l}(x) \equiv P_{l}^{0}(x) .
$$

The orthonormality relationship for associated Legendre functions is given by

$$
\int_{-1}^{1} d x P_{l}^{m}(x) P_{l^{\prime}}^{m}(x)=\delta_{l l^{\prime}} \frac{2}{2 l+1} \frac{(l+m) !}{(l-m) !} .
$$

These functions satisfy the following recursion relations (see Eqs. 8.733 in Ref. [48]):

$$
\begin{gathered}
P_{l}^{m+2}(x)+2(m+1) \frac{x}{\sqrt{1-x^{2}}} P_{l}^{m+1}(x) \\
+(l-m)(l+m+1) P_{l}^{m}(x)=0,
\end{gathered}
$$

$$
\begin{aligned}
& (2 l+1) \sqrt{1-x^{2}} P_{l}^{m-1}(x)=P_{l-1}^{m}(x)-P_{l+1}^{m}(x), \\
& (2 l+1) x P_{l}^{m}=(l-m+1) P_{l+1}^{m}+(l+m) P_{l-1}^{m}, \\
& \left(1-x^{2}\right) \frac{d P_{l}^{m}}{d x}=(m+1) x P_{l}^{m}-(l-m+1) P_{l+1}^{m},
\end{aligned}
$$




$$
\left(1-x^{2}\right) \frac{d P_{l}^{m}}{d x}=-l x P_{l}^{m}+(l+m) P_{l-1}^{m} .
$$

Using orthonormality and completeness of Legendre polynomials, an associated Legendre function of $m=2$ can be written in terms of Legendre polynomials using the integral [49]

$$
\begin{aligned}
& \int_{-1}^{1} d x P_{l}(x) P_{j}^{2}(x) \\
& \quad= \begin{cases}0, & l>j \text { or } j+l \text { odd }, \\
-2 j(j-1) /(2 j+1), & j=l, \\
4, & l<j \text { and } j+l \text { even. }\end{cases}
\end{aligned}
$$

With the above conventions for Legendre functions, the spherical harmonics are given by

$$
Y_{(l m)}(\theta, \phi)=\sqrt{\frac{2 l+1}{4 \pi} \frac{(l-m) !}{(l+m) !}} P_{l}^{m}(\cos \theta) e^{i m \phi} .
$$

The convolution of a Legendre polynomial with a spherical harmonic is

$$
\int d \hat{\mathbf{n}} P_{j}(\hat{\mathbf{k}} \cdot \hat{\mathbf{n}}) Y_{(l m)}(\hat{\mathbf{n}})=\frac{4 \pi}{2 l+1} Y_{(l m)}(\hat{\mathbf{k}}) \delta_{l j}
$$

This can be obtained by expressing the Legendre polynomial in terms of spherical harmonics with the spherical harmonic addition theorem and then using the orthonormality of spherical harmonics.

Angular-momentum lowering and raising operators can be used to derive the following recursion relations for spherical harmonics:

$$
\begin{aligned}
e^{i \phi} \sin \theta Y_{(l m)}= & -\left[\frac{(l+m+1)(l+m+2)}{(2 l+1)(2 l+3)}\right]^{1 / 2} Y_{(l+1, m+1)} \\
& +\left[\frac{(l-m)(l-m-1)}{(2 l-1)(2 l+1)}\right]^{1 / 2} Y_{(l-1, m+1)}, \\
e^{-i \phi} \sin \theta Y_{(l m)}= & {\left[\frac{(l-m+1)(l-m+2)}{(2 l+1)(2 l+3)}\right]^{1 / 2} Y_{(l+1, m-1)} } \\
& -\left[\frac{(l+m)(l+m-1)}{(2 l-1)(2 l+1)}\right]^{1 / 2} Y_{(l-1, m-1)},
\end{aligned}
$$

which can be iterated and evaluated for $m=0$ to obtain $\cos 2 \phi \sin ^{2} \theta Y_{(l 0)}$

$$
\begin{aligned}
= & \frac{1}{2}\left\{[ \frac { ( l + 1 ) ( l + 2 ) ( l + 3 ) ( l + 4 ) } { ( 2 l + 1 ) ( 2 l + 3 ) ^ { 2 } ( 2 l + 5 ) } ] ^ { 1 / 2 } \left(Y_{(l+2,2)}\right.\right. \\
& \left.+Y_{(l+2,-2)}\right)-2 \frac{\sqrt{l(l-1)(l+1)(l+2)}}{(2 l-1)(2 l+3)}\left(Y_{(l, 2)}+Y_{(l,-2)}\right) \\
& \left.+\left[\frac{l(l-1)(l-2)(l-3)}{(2 l-3)(2 l-1)^{2}(2 l+1)}\right]^{1 / 2}\left(Y_{(l-2,2)}+Y_{(l-2,-2)}\right)\right\} .
\end{aligned}
$$

For the replacement $\cos 2 \phi \rightarrow \sin 2 \phi$ make the replacements $(1 / 2) \rightarrow(1 / 2 i)$ in the prefactor and $\left(Y_{(x, 2)}\right.$ $\left.+Y_{(x,-2)}\right) \rightarrow\left(Y_{(x, 2)}-Y_{(x,-2)}\right)$.

Looking at the $\theta$ dependence of Eq. (B15), we get

$$
\begin{aligned}
\left(1-x^{2}\right) P_{j}(x)= & \frac{P_{j+2}^{2}}{(2 j+1)(2 j+3)}-\frac{2 P_{j}^{2}}{(2 j-1)(2 j+3)} \\
& +\frac{P_{j-2}^{2}}{(2 j+1)(2 j-1)} .
\end{aligned}
$$

[1] For a review of CMB measurements through the end of 1994, see M. White, D. Scott, and J. Silk, Annu. Rev. Astron. Astrophys. 32, 329 (1994).

[2] See the MAP home page, URL http://map.gsfc.nasa.gov

[3] See the Planck home page, URL http://astro.estec.esa.nl/SAgeneral/Projects/Cobras/cobras.html

[4] J. R. Bond et al., Phys. Rev. Lett. 72, 13 (1994).

[5] W. Hu and N. Sugiyama, Phys. Rev. D 51, 2599 (1995); W. $\mathrm{Hu}$ and N. Sugiyama, Astrophys. J. 444, 489 (1995); W. Hu and M. White, ibid. 471, 30 (1996).

[6] M. Kamionkowski, D. N. Spergel, and N. Sugiyama, Astrophys. J. Lett. 426, L57 (1994).

[7] G. Jungman, M. Kamionkowski, A. Kosowsky, and D. N. Spergel, Phys. Rev. Lett. 76, 1007 (1996); Phys. Rev. D 54, 1332 (1996).

[8] J. Magueijo and M. P. Hobson, Report No. astro-ph/9610105 (unpublished).

[9] E. Gates, S. Dodelson, and A. Stebbins, Astrophys. J. 467, 10 (1996).
[10] J. R. Bond and G. Efstathiou, Astrophys. J. Lett. 285, L47 (1984); Mon. Not. R. Astron. Soc. 226, 655 (1987).

[11] M. Zaldarriaga and D. Harari, Phys. Rev. D 52, 3276 (1995).

[12] R. A. Frewin, A. G. Polnarev, and P. Coles, Mon. Not. R. Astron. Soc. 266, L21 (1994); D. Harari and M. Zaldarriaga, Phys. Lett. B 319, 96 (1993); K. L. Ng and K. W. Ng, Astrophys. J. 445, 521 (1995); E. Milaneschi and R. Valdarnini, Astron. Astrophys. 162, 5 (1986); R. G. Crittenden, R. L. Davis, and P. J. Steinhardt, Astrophys. J. 417, L13 (1993).

[13] A. Kosowsky, Ann. Phys. (N.Y.) 246, 49 (1996).

[14] D. Coulson, R. Crittenden, and N. G. Turok, Phys. Rev. Lett. 73, 2390 (1994); A. Melchiorri and N. Vittorio, in The Cosmic Background Radiation (CBR 96), Proceedings of the NATO Advanced Study Institute, Strasbourg, France, 1996 (Plenum, New York, in press).

[15] R. K. Sachs and A. M. Wolfe, Astrophys. J. 147, 73 (1967); M. White and W. Hu, Report No. astro-ph/9609105 (unpublished). 
[16] R. Crittenden, D. Coulson, and N. G. Turok, Phys. Rev. D 52, 5402 (1995).

[17] A. Kosowsky and A. Loeb, Astrophys. J. 469, 1 (1996).

[18] M. Zaldarriaga, Phys. Rev. D 55, 1822 (1997); K. L Ng and K. W. Ng, ibid. 51, 364 (1995); Astrophys. J. 456, 413 (1996).

[19] M. Kamionkowski, A. Kosowsky, and A. Stebbins, Phys. Rev. Lett. 78, 2058 (1997).

[20] U. Seljak and M. Zaldarriaga, Phys. Rev. Lett. 78, 2054 (1997).

[21] U. Seljak and M. Zaldarriaga, Phys. Rev. D 55, 1830 (1997).

[22] For a review, see J. E. Lidsey et al., Report No. astro-ph/9508078 (unpublished).

[23] E. J. Wollack et al., Astrophys. J. 419, L49 (1993); P. M. Lubin, P. Melese and G. F. Smoot, ibid. 273, L51 (1983); R. B. Partridge, J. Nowakowski, and H. M. Martin, Nature (London) 331, 146 (1988).

[24] B. Keating, A. Polnarev, J. Steinberger, and P. Timbie (in preparation); F. Melchiorri, C. Montecchio, G. Pisano, and B. Olivo, in The Cosmic Background Radiation (CBR 96) [14], Proceedings of the NATO/ASI School, Strasbourg, 1996 (unpublished).

[25] E. Newman and R. Penrose, J. Math. Phys. (N.Y.) 7, 863 (1966); J. N. Goldberg et al., ibid. 8, 2155 (1967).

[26] See, e.g., J. D. Jackson, Classical Electrodynamics, 2nd ed. (Wiley, New York, 1975) or M. Born and E. Wolf, Principles of Optics, 6th ed. (Pergamon, New York, 1980).

[27] S. Chandrasekhar, Radiative Transfer (Dover, New York, 1960), Chap. 1.

[28] A. Stebbins, Astrophys. J. (to be published).

[29] K. S. Thorne, Rev. Mod. Phys. 52, 299 (1980).

[30] F. J. Zerilli, J. Math. Phys. (N.Y.) 7, 2203 (1970).
[31] M. Kamionkowski, A. Kosowsky, and A. Stebbins (in preparation).

[32] L. Knox, Phys. Rev. D 52, 4307 (1995).

[33] R. A. Fisher, J. R. Stat. Soc. 98, 39 (1935).

[34] M. Kamionkowski and A. Kosowsky (in preparation).

[35] Some recent codes are described in N. Sugiyama and N. Gouda, Prog. Theor. Phys. 88, 803 (1992); J. R. Bond et al., Phys. Rev. Lett. 72, 13 (1994); C. P. Ma and E. Bertschinger, Astrophys. J. 455, 7 (1995); R. Stompor, Astron. Astrophys. 287, 693 (1994); W. Hu et al., Phys. Rev. D 52, 5498 (1995).

[36] A. G. Polnarev, Sov. Astron. 29, 607 (1985).

[37] L. F. Abbott and M. Wise, Nucl. Phys. B244, 541 (1984).

[38] U. Seljak and M. Zaldarriaga, Astrophys. J. 469, 437 (1996).

[39] U. Seljak, Report No. astro-ph/9608131 (unpublished).

[40] N. Kaiser, Astrophys. J. 388, 272 (1992).

[41] J. C. R. Magueijo, Phys. Rev. D 49, 671 (1994); D. Harari, J. Hayward, and M. Zaldarriaga, ibid. 55, 1841 (1997).

[42] A. Kosowsky and A. Loeb (in preparation).

[43] R. Sunyaev and Ya. B. Zeldovich, Annu. Rev. Astron. Astrophys. 18, 537 (1980).

[44] M. Kamionkowski and A. Loeb, Report No. astro-ph/9703118 (unpublished).

[45] S. Cortiglioni and T. A. Spoelstra, Astron. Astrophys. 302, 1 (1995).

[46] A. Franceschini, Astrophys. J. 344, 35 (1989); Astron. Astrophys. Suppl. Ser. 89, 285 (1991).

[47] W. N. Brandt et al., Astrophys. J. 424, 1 (1994); S. Dodelson and A. Stebbins, ibid. 433, 440 (1994); S. Dodelson, Report No. astro-ph/9512021 (unpublished); M. Tegmark and G. Efstathiou, Mon. Not. R. Astron. Soc. 281, 1297 (1996).

[48] I. S. Gradshteyn and I. M. Ryzhik, Tables of Integrals, Series, and Products (Academic, San Diego, 1980).

[49] D. Coulson, Ph.D. thesis, Princeton University, 1994. 